DOES TIME HEAL ALL WOUNDS?:

THE CHANGING MENTAL HEALTH OF REFUGEES OVER TIME

\author{
by \\ Stephanie Nicole Fraser \\ B.A. Honours, Queen's University, 2013 \\ A Major Research Paper \\ presented to Ryerson University
}

In partial fulfillment of the requirements for the degree of

\author{
Master of Arts \\ in the Program of \\ Immigration and Settlement Studies
}

Toronto, Ontario, Canada, 2017

(C) Stephanie Nicole Fraser, 2017 


\section{AUTHOR'S DECLARATION FOR ELECTRONIC SUBMISSION OF A MAJOR RESEARCH PAPER (MRP)}

I hereby declare that I am the sole author of this Major Research Paper (MRP). This is a true copy of the MRP, including any required final revisions.

I authorize Ryerson University to lend this MRP to other institutions or individuals for the purpose of scholarly research

I further authorize Ryerson University to reproduce this MRP by photocopying or by other means, in total or in part, at the request of other institutions or individuals for the purpose of scholarly research.

I understand that my MRP may be made electronically available to the public.

Stephanie Nicole Fraser 


\title{
DOES TIME HEAL ALL WOUNDS?: \\ THE CHANGING MENTAL HEALTH OF REFUGEES OVER TIME
}

(C) Stephanie Nicole Fraser

Master of Arts 2017

Immigration and Settlement Studies

Ryerson University

\begin{abstract}
An increase of ongoing armed conflicts has resulted in substantial numbers of refugees around the world. The traumatic experiences refugees face can be detrimental to their mental health, further compounded by resettlement stressors upon arrival in Canada. This qualitative study incorporates an intersectional social determinants of health framework in order to understand the changes in mental health of refugees at different stages of a lifelong (re)settlement process. The findings of this study are informed by interviews with five service providers. Results indicated a number of salient post-migration factors that influence mental health in both the short and over the longer term for refugees and further elucidated the effects of a mutually-reinforcing relationship between resettlement stressors and trauma in mental health changes. Implications of the study findings reveal a critical need for a more psychosocial approach to be taken regarding refugee mental healthcare in future research as well as psychological interventions.
\end{abstract}

Key Words: Intersectionality; mental health; refugees; resettlement; service provision; social determinants of health; trauma 


\section{ACKNOWLEDGEMENTS}

I am very fortunate to have been guided by countless people in rich and diverse ways throughout my educational journey. First, to my supervisor Dr. Sepali Guruge, for her support, guidance, and tenacity in the writing of this project. Also thank you to Dr. Cristina Catallo for her genuine interest and helpful feedback as my second reader.

To my wonderful ISS cohort - thank you for the support, the laughs, the tears, the witty banter, and the conversations that have forever changed the way I reflect on and experience the world around me. Although we only met a short year ago, many of you will remain my friends for the rest of my life, and for that I am truly thankful.

To all the incredible participants who dedicate their lives to helping people in need and allowed me to learn from their wisdom and strength. This project would not have been possible without their involvement, their honesty, and their voices.

The unconditional love and support of my family are the foundation of all my achievements, and I am grateful for them every day. Mom, Dad, and Grant - thank you from the bottom of my heart. All the best parts of me have come from each of you.

Finally, to Jason. Thank you for your love, for encouraging me to pursue my dreams even though it meant having a country between us, for reading every single essay, and for being my biggest supporter without hesitation. Most of all, thank you for listening to everything. 


\section{TABLE OF CONTENTS}

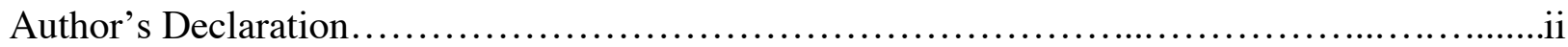

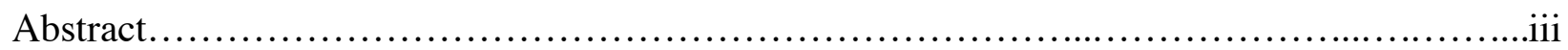

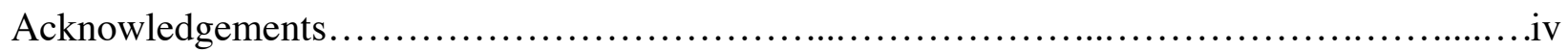

\section{Chapter 1: Introduction}

1.1 Research Background............................................................ 1

1.2 Purpose and Significance of the Study ........................................... 4

1.3 Structure of the Major Research Paper....................................................

\section{Chapter 2: Review of the Literature}

2.1 An Overview of Refugee Mental Health.........................................6

2.2 Psychological Stages of the Refugee Process....................................... 10

2.3 The Impact of Resettlement Stressors................................................. 14

2.4 The Relationship Between Time and Mental Health................................ 17

2.5 Perspectives on Mental Health Service Provision................................... 21

\section{Chapter 3: Theoretical Frameworks}

3.1 Social Determinants of Mental Health......................................... 27

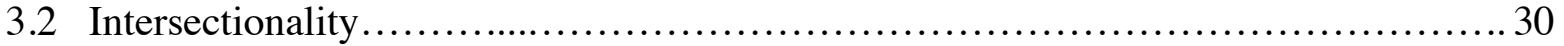

\section{Chapter 4: Methodology}

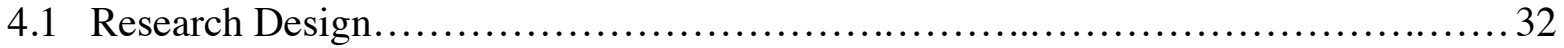

4.2 Sampling Technique......................................................... 33

4.3 Ethical Considerations............................................................. 34

4.4 Recruitment Strategy and Participant Profile......................................... 35

4.5 Data Collection.............................................................. 36

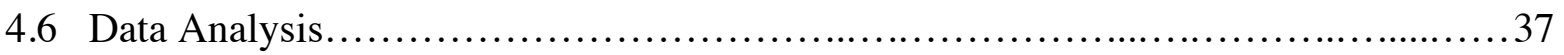

\section{Chapter 5: Findings}

5.1 Client Demographics...................................................... 39

5.2 Resettlement Stressors ..................................................... 40

5.3 Barriers to Accessing Services............................................. 52

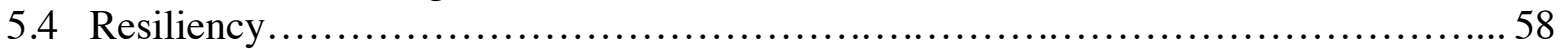

5.5 Changes in Mental Health Over Time ..................................................................59

\section{Chapter 6: Discussion and Conclusion}

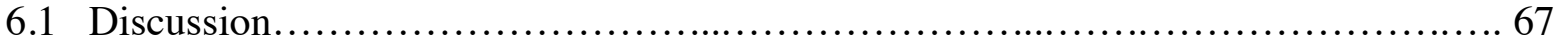

6.2 Limitations and Recommendations for Future Research........................... 77

6.4 Conclusion............................................................... 79 


\section{Appendices}

Appendix A: Interview Guide.......................................................... 80

Appendix B: Participant Consent Form.............................................. 81

References ...................................................................................................................................................... 85 
It is as though, amidst the peril and pain of refugee life and experience, the less obvious and long-term toll that is exacted on mental health and well-being must be endured and tolerated. Yet, to all those working with refugees, it is obvious that the psychological and emotional scars associated with the refugee experience remain as a constant and abiding source of terror, humiliation, and indignity (Marsella, Bornemann, Ekblad, \& Orley, 1994, p. 6).

\section{CHAPTER 1: INTRODUCTION}

\subsection{Research Background}

Mental health is integral to overall human health and is defined by the World Health Organization (WHO, 2010) as, "a state of well-being in which an individual realizes his or her own abilities, can cope with the normal stresses of life, can work productively, and is able to make a contribution to his or her community." While mental health is recognized to be affected by some biological factors, it is determined and affected to a greater extent by numerous social and psychological components, such as the often daily stressful, discriminatory, and violent phenomena experienced by those living in horrific circumstances where their basic human rights are threatened (Elliot \& Segal, 2012).

Although forced migration existed long before, since 1945 a dramatic increase in ongoing armed conflicts, both within and between nations, has resulted in substantial numbers of asylum seekers and refugees (Ingleby, 2004). This epidemic of violence that has inflicted human-engendered trauma on populations residing in numerous regions of the world has forcibly displaced nearly 21.3 million refugees globally (UNHCR, 2016). From a human rights perspective, the current international refugee situation continues to be a monumental challenge to those from a vast range of disciplines who work to preserve and improve the mental health and well-being of refugees (Marsella et al., 1994). Canada, as a country committed to fulfilling its humanitarian obligation of providing (re)settlement, has one of the largest populations of 
(re)settled refugees in the world whereby individuals selected by the government and private sponsorship are granted refugee status abroad and given landed residency upon arrival (Lacroix, 2004). They may also arrive in Canada and claim asylum once in the country, having to undergo the refugee determination process, often a traumatic and stressful event in itself as the already vulnerable claimant must prove that their story is credible and that they are accordingly worthy of humanitarian protection (Lacroix, 2004). As defined by the 1951 United Nations Convention Relating to the Status of Refugees, a refugee is someone who is "unable or unwilling to return to their country of origin owing to a well-founded fear of being persecuted for reasons of race, religion, nationality, membership of a particular social group or political opinion" (Citizenship and Immigration Canada, 2013). Many governments have further expanded this definition in an effort to recognize refugees as "people who are not targets of persecution, but who are victims of war, violence, and other social and political disasters (Marsella et al., 1994, p. 3). As traumatic experiences that they face are unlikely to be single events but are instead cumulative and interrelated, often shattering their families and sense of home (Schweitzer, Melville, Steel, \& Lacherez, 2006), it is not surprising that a large body of scholarship demonstrates that forced migration is detrimental to the mental health of refugees. The physical and psychological distress that refugees experience during the pre-migration and migration stages is compounded by the difficulties and fears that they face during the stressful and often re-traumatizing process of (re)settlement (Miller \& Rasmussen, 2010; Beiser \& Hyman, 1997; Carswell, Blackburn, \& Barker, 2011).

Working with refugees presents mental health professionals with a unique set of challenges based upon the individual experiences, such as armed conflict, torture, persecution, and other attendant violations of human rights refugees may have encountered prior to 
dislocation (Murray, Davidson, and Schweitzer, 2010). Also of importance to note is that, because the refugee community reflects the continually shifting distribution of violent conflict throughout the world, the demographics of refugees in countries of resettlement such as Canada are also in constant flux.

Considerable research and subsequent interventions focussing on refugee mental health have proceeded from a biomedical perspective that proposes a unidirectional 'dose-response' relationship in which psychiatric symptoms are understood as an almost automatic and immediate reaction to a single pathogen, attributed to be the various types of trauma that many refugees have faced (Porter \& Haslam, 2005). Simich, Este, and Hamilton (2010) claim that, from the 1980s onwards, the dominant discourse of pathologizing refugees has downplayed their psychological and social sources of resiliency, painting them as passive victims of psychological distress and mental illness such as Post Traumatic Stress Disorder (PTSD). The predominant result of fixating on the overriding trauma centred approach within the refugee mental health field is that it has become self-perpetuating and, as such, "criticism of the one-sidedness of this approach was routinely waved aside as callous indifference to the plight of those suffering" (Ingleby, 2004, p. 9). However, this model has been contested in more recent years based upon its failure to adequately portray all dimensions of the complex refugee experience (e.g. Hollifield, Warner, Lian, et al., 2002).

In light of contemporary research that presents a more comprehensive understanding that captures not only the disconcerting and often horrific experiences this population have encountered, but also the acculturation shock and daily stressors they continue to face upon arrival in the country of resettlement, many countries have begun to recognize the struggles they face in meeting the mental health needs of the refugee population (George, 2012). As a result, 
there has been a noticeable shift away from the primary focus on researching and implementing mental health interventions for refugees based on patterns of psychiatric symptomatology (Miller $\&$ Rasco, 2004) towards a paradigm of mental health that works to identify interacting postmigration social determinants of mental health (Beiser, 2005; Porter \& Haslam, 2005).

Although time can be a powerful healer, the relationship between early and later psychological symptomatology comprises a complex interplay between numerous determinants and intersecting components of identity, which is often mediated by refugees' incredible resiliency. There is a frequent disconnect between those conducting research on refugee mental health and those providing psychological services to this population. Thus, by incorporating the theoretical frameworks of social determinants of health and intersectionality, this research provides a unique account from the perspective of the service provider as to how the mental health trajectories of refugees are multifaceted psychological processes that likely require distinct needs at different stages of a refugee's lifelong (re)settlement process. This study also examines the types of suggestions put forth by mental health care professionals in order to improve services for the refugee population in Canada.

\subsection{Purpose and Significance of the Study}

It is important to evaluate the fluctuating and long-term effects of both pre-migration trauma and post-migration stressors that refugees experience in order to improve the mental health services that are offered in Canada. The purpose of this research is to explore from the perspective of healthcare professionals how the mental health of refugees changes over time, and which resettlement stressors exert the most influence on mental well-being. This study contributes to the large body of existing scholarship on refugee mental health by examining how mental health service needs of this population are flexible and subject to change over the course 
of their resettlement process due to the intersection of numerous, complex pre- and postmigratory determinants.

As will be expanded upon in the literature review, there is a paucity of research that examines how the mental health needs of refugees change over time, particularly from the perspective of service providers. In order to gain a better understanding of the interaction between pre-migration trauma and post-migration settlement experiences of refugees, the following research questions guided this study:

(1) What resettlement stressors have the most influence on refugee mental health?

(2) How does the mental health of refugees change over time?

\subsection{Structure of the Major Research Paper}

This Major Research Paper (MRP) will explore the proposed research questions through qualitative, semi-structured interviews with mental healthcare professionals that work with refugees in the Greater Toronto Area. This MRP is comprised of six main chapters which are subdivided into distinct sections. First, an extensive review of the existing literature regarding refugee mental health will be presented in Chapter 2 in order to introduce the context within which the study purpose and research questions have been developed. Chapter 3 presents a brief introduction to the complementary theoretical frameworks of social determinants of health and intersectionality that underpin the interpretation of findings. The methodology utilized for this study is subsequently described in Chapter 4 in terms of research design, sampling, recruitment and participants, data collection, and the method of analysis employed to extract themes from the data. Findings from participant interviews and an examination of responses are then covered in Chapter 5, while the $6^{\text {th }}$ and final chapter puts forth a discussion of the results as well as study limitations and recommendations for future research. 


\section{CHAPTER 2: REVIEW OF THE LITERATURE}

\subsection{An Overview of Refugee Mental Health}

The cornerstone of being a refugee is characterized by adverse, sequential events that do not end with forced displacement, but are instead only the beginning of a long period of turbulence and uncertainty (Fazel, Reed, Panter-Brick, \& Stein, 2012). Through the process of leaving behind the familiarity of home and the supports provided by familial and other social networks, a complex interrelationship between exposure to cumulative traumas and stressors develop that pose a major threat to physical, economic, and psychological welfare. For refugees, the process of migration is exceptionally heterogeneous, comprised of a myriad of experiences and reasons for displacement characterized by a breakdown of social and cultural structures, systematic violations, and the burden of short and long term adaptation to refugee camps or novel places of resettlement. Thus, not all refugee communities are likely to undergo the same or even similar experiences both prior to and after migrating and, therefore, forced relocation is incredibly nuanced for each individual depending on factors such as gender, age, country of origin, types of trauma experienced, among others. As a consequence, the psychological effects and implications for individual refugees that are a by-product of these difficult and often prolonged situations are highly variable (Bhugra, 2004). Moreover, there is evidence that female gender and older age are risk factors for increased psychological distress, including comorbid symptoms of PTSD, depression, and anxiety, highlighting the need for an intersectional approach to both research and the provision of mental health services (Porter \& Haslam, 2005; Schweitzer et al., 2006; Haldane \& Nickerson, 2016). However, Marsella et al. (1994) claim that because governmental and nongovernmental efforts are more likely directed at the priorities of basic 
human survival, such as language acquisition and obtaining employment, the immediacy of these needs have relegated mental health to a position of lesser concern.

The refugee experience, as mentioned, is a highly individual experience, but is generally agreed upon in migration literature as proceeding through three phases. All stages are germane to the topic of traumatic experiences displaced persons undergo, consisting of pre-migration, migration, and post-migration. Each of these are distinct and characterized by their own set of life chances, challenges, and potential for harrowing situations to occur due to the deprivation of relocation. During pre-migration refugees may encounter, either directly or indirectly, gross human rights violations such as sexual assault, torture, violence, death of family members, and an overall heightened and unrelenting fear for their lives. Length of time and conditions living in refugee camps as well as residing in another country before permanent resettlement are added factors that may occur before flight takes place, and resultantly need to be acknowledged in the context of refugee mental health. As noted by 2015 data, before arrival in a country of resettlement and protection, two-thirds of the international refugee population live in protracted situations in refugee camps, which is typically a site of intense stress, uncertainty and fear (Wessels, 2014). The second stage of migration begins when refugees initially flee their home across an international border, oftentimes accomplished through treacherous means such as by boat or the use of smugglers. During this transition, refugee may be separated from family members, face exhaustion and starvation from frequent traveling, and worry about detention or imprisonment upon arrival in their destination (Hodes, 2000). While both pre-migration and migration entail intense, multiple-event traumas that increase the potential for lasting psychological impacts, these experiences are further compounded by stressors that are faced by refugees upon arrival in a new country related to resettlement and acculturative related 
challenges, which may jeopardize their existing sense of empowerment, identity, and overall life meaning (Schweitzer et al., 2006).

Investigations since the 1980s examining the effects of trauma on the development of psychopathology in post-migration has documented a robust unidirectional dose-response correlation between traumatic events and mental health issues. This association has emerged from studies in which cumulative trauma has been linked to an increased risk of the manifestation of psychiatric morbidity, relying on quantitative self-reported data that has arisen from measures such as the Harvard Trauma Questionnaire (e.g., Chung \& Kagawa-Singer, 1993; Mollica, McInnes, Pool, \& Tor, 1998). A systematic review by Fazel, Wheeler, and Danesh (2007) of 7,000 refugees resettled in seven Western countries revealed that $9 \%$ and $11 \%$ of refugee adults and children, respectively, reported symptoms of PTSD - approximately ten times the prevalence rate for the general population. These findings are congruent with a study that examined the long-term effects of psychological trauma on the mental health outcomes of Vietnamese refugees resettled in Australia (Steel, Silove, Phan, \& Bauman, 2002). The authors asked respondents to indicate types of trauma they had been exposed to out of 24 categories such as, "witnessing someone being badly injured or killed", "being close to death", and "imprisonment". In sum, this research established a dose-response relationship between cumulative trauma and psychiatric symptomatology, as respondents who had been exposed to three or more trauma categories had an eight-fold increase in mental ill health risk as opposed to those with no exposure (Steel et al., 2002). However, other literature has contested the doseresponse correlation by arguing that these studies over-estimate the magnitude of the direct effect of pre-migration traumas, which cannot simply be added up but must be considered in the context of a refugee's entire experience including personal history and the interplay of other 
factors that may influence mental health in the period of post-migration (e.g., Kessler et al., 2010; Fazel et al., 2012; Beiser \& Hou, 2014).

While trauma-centred research has dominated refugee mental health studies since the beginning of this field, more contemporary scholars have challenged the linear dose-response paradigm by proposing other components that affect mental health. Bhugra (2004), for instance, claims that two key factors in the genesis of stress of the refugee process that affects psychological outcomes later on are the geographic distance that must be traversed to the country of resettlement and the amount of preparation given prior to migration. Similarly, scholarship by Keller (1975, as cited in George, 2012) suggests that the trauma of migration produces residual psychological states that affect mental health for many years to come; however, he argues that different typologies of flight and settlement affect psychological outcomes in dissimilar ways.

A study by George (2012) offers a preliminary attempt to test this notion and looked specifically at the interaction between typologies of refugees based on the characteristics of their migration (acute versus anticipatory), typology of settlement (new versus traditional) and psychological distress. Whereas acute refugees are those forced to flee their home country within a few days or even hours due to a range of potentially life threatening disasters, anticipatory refugees are aware of the need to leave their home country before the present situation escalates, typically with their personal resources and families intact (George, 2012). In terms of settlement, 'new' refugees are "culturally, racially, and ethnically vastly different from their hosts, and are likely to lack kin or potential support groups" whereas 'traditional' refugees are culturally and ethnically similar to the host population (George, 2012, p. 432). Results from this research suggest that new acute refugees exhibit the highest levels of distress later on due to the greater amount of trauma experienced prior to and during migration which compounds with a lack of 
personal resources and social support upon arrival in the country of resettlement. Cross-cultural analysis such as this is beneficial to further solidifying a departure from the fully biomedical model of trauma that has, up until recently, principally guided research and interventions regarding refugee mental health. These studies additionally provide a multilevel understanding of refugee trauma as influenced by numerous determinants which must be considered in policy design and the provision of services.

\subsection{Psychological Stages of the Refugee Process}

In an effort to conceptualize the psychological changes sojourners undergo and provide both researchers and practitioners with a greater understanding of how migration and resettlement affects mental health, Grove and Torbiorn (1985) developed a theoretical model with the intent of going beyond "the symptom level" and uncovering "consistent explanation[s] of the person's 'inner world' of basic cognitive and emotional processes” (p. 206). Although their model serves to encapsulate the experiences of all categories of sojourners, Gonsalves (1992) contends that the compulsory and undoubtedly traumatic nature of migration specific to the plight of refugees situates these communities in a uniquely vulnerable position at risk of potentially severe psychological distress. While it has been noted that the refugee experience is a multi-faceted and highly individual process that produces mental health effects which are dependent on a multiplicity of variable factors, Gonsalves (1992) modified Grove and Torbiorn's (1985) original framework specifically to the experiences of refugees, positing that they progress through a series of psychological stages upon arrival and throughout resettlement in a host country. As such, this model is important to highlight because it is the only paradigm that views the refugee process and its psychological variability as a function of time, entailing five distinctive stages of resettlement that combine with other social factors, such as gender, 
personality, impact of pre-flight and flight trauma, and settlement stressors. Whereas Gonsalves (1992) acknowledges that it is unlikely for individuals to procede through the proposed stages as smoothly or even possibly in the same order that he has delineated, the primary aim in the development of this framework is to guide how mental health providers assist refugees not only upon initial arrival, but throughout their active and lifelong processes of resettlement.

This framework proposes that during the first stage, early arrival, refugees "arrive at a host country with a variety of behavioural and affective responses" (Gonsalves, 1992, p. 384). Consequently, in approximately the first six months, they feel and display a range of emotions, from relief, positivity, and excitement about beginning a new life to confusion, guilt about leaving behind others (i.e. friends and family members) who continue to suffer, and utter despair. It is not uncommon for refugees to live in the country of resettlement during this stage "almost as though they were still in their own homeland" (Gonsalves, 1992, p. 384) as they have not yet come to terms with the loss and trauma they have experienced prior to migration or felt estrangement from the host country. According to Gonsalves (1992), the role of mental health services providers in this stage should be orienting refugees to their new homes and providing a culturally appropriate bridge for the transition between life in the country in which they left and the country of resettlement.

Stage two is labelled destabilization, lasting from approximately six months to three years, often experienced as extremely stressful and painful due to acculturation challenges. This phase is characterized by upset and crises as refugees attempt to become oriented to and make sense of their new home. Refugees may feel anger and hostility as they resist conforming to a novel culture and are simultaneously psychologically preoccupied by stressors of surviving economically, finding employment, securing housing, orienting to the new country through 
cultural learning, and dealing with changes in family and partner dynamics. Of significance, Gonsalves (1992) maintains that during this time, mental health services and psychotherapeutic interventions may be resisted by refugees due to the fact that "the amount of time and energy demanded in meeting survival needs and the resulting tendency to postpone dealing with other, possibly more painful issues" (p. 385). Therefore, even those in the most serious psychological distress are unlikely to want or seek help due to denial or a postponement of issues. Refugees may additionally have difficulty establishing a frame of reference for themselves and are "filled with pervasive ambiguity" (Gonsalves, 1992, p. 382). As refugees struggle to find their place in the new society, they often become vulnerable to past memories; thus, sadness and nostalgia for the life they left behind may ensue, particularly if they encounter barriers to employment and inclusion in the social fabric of everyday life.

Gonsalves (1992) denotes the stage from three to five years as experimentation and stabilization due to refugees' tendency to experiment with differential cultural learning strategies that characterize this phase. Most evident is a behavioural learning curve as refugees undertake 'trial and error' behaviours to find their sense of identity and place within the new society, typically after they have obtained employment and are, for the most part, stabilized in their basic needs. Conflict, such as marital issues and child-rearing problems, may occur due to friction between their culture and the North American mainstream, and therefore anxiety is also highly prevalent.

During stage four, a return to normal life, the originally exciting, overwhelming, and potentially threatening host country "becomes a routine backdrop for daily life" (Gonsalves, 1992, p. 387). At this point, refugees have been living in the host country for approximately five to seven years and, as such, they have typically selected the particular values of the native 
culture they wish to retain and negotiated an 'identity foreclosure' with the mainstream culture. However, this stage presents new potentially psychologically harmful challenges as they try to cope with personality changes that have resulted from the refugee experience as well as the possible resurfacing of a delayed grief reaction. As Gonsalves (1992) summarizes, the "[r]elative economic stability may free them to confront the painful feelings and memories that were suppressed in the service of learning to live in a new environment" (p. 387). Psychological issues that were postponed in the service of economic and social adaptation such as depression, PTSD, anxiety, or a comorbidity of symptoms may occur. Service providers must be aware of the possibility that past losses, guilt, and anger may not have been previously worked through and tend to appear during this stage in the form of risk taking, outbursts, and survivor's guilt regarding those that are still caught in areas of dire conflict and violence.

Finally, the last stage is decompensation, which can actually occur in conjuncture with any other stage and is characterized by feeling overwhelmed with the demands of reality. Refugees may experience a 'precipitous collapse' of their ability to cope successfully. While Gonsalves (1992) maintains that very few refugees decompensate completely, most do to an extent at some point. This serious symptomatology is underpinned by both psychological and environmental issues. For example, refugees may feel pulled apart by the extreme changes they go through during the processes of migration and resettlement. Similarly, leaving family members or friends behind in a country of war or conflict, failure to obtain desired employment, and marital or intergenerational conflict are all circumstances that may, in a few individual cases, trigger extreme psychiatric symptoms such as schizophrenia, major depressive disorder (MDD), or abuse of drugs and alcohol. 
While Gonsalves' (1992) appended theoretical model posits relationships between preand post-migration exposures and outcomes, LeMaster et al. (2017) highlight that his proposed framework has not been empirically tested, suggesting the need for longitudinal studies that elucidate "temporal processes in refugee health before and after migration, and how the relationship between pre- and post-migration stressors affects subsequent acculturation and mental health" (p. 2). These scholars sought to be the first to test the stage model proposed by Grove and Torbiorn (1985) and Gonsalves (1992) by measuring multiple potential factors in both the pre- and post-migration periods and outcomes relevant to a cohort of refugees who had recently arrived in the United States at baseline and one year later. Interestingly, this study found that trauma experienced in pre-migration predicted less acculturation one year after resettlement and only predicted refugees' symptoms of PTSD indirectly. These findings suggest that the relationship between trauma and later symptomatology, rather than being straightforward, is mediated by other factors in the resettlement environment such as social support, maladaptive (e.g., denial, social isolation, and self-blame) or adaptive coping mechanisms, and economic growth. This study advances the literature by showcasing that although pre-migration characteristics such as trauma influence refugees' mental health in the country of resettlement, multilevel post-migration determinants like acculturation stress, social support, unemployment or underemployment, the availability of appropriate services, and cultural bereavement are likely to have an even greater negative affect on mental health from a long term perspective (LeMaster et al., 2017).

\subsection{The Impact of Resettlement Stressors}

Subject to extensive debate, a central issue in refugee mental health research has been if psychological reactions to trauma persist over time or if they occur acutely and are thereby likely 
to be resolved shortly upon resettlement once traumatic situations have been removed and physical safety is restored. While it has been claimed that an exaggeration of the mental health effect of trauma risks medicalizing and stigmatizing refugees by overlooking their resilience and ability to cope, an underestimation of the mental health consequences of the deprivation experienced in the post-migration setting leads to a potential disregard and neglect of their genuine mental health service needs (Steel, Silove, Phan, \& Bauman, 2002).

With this in mind, it is important to acknowledge the extent to which circumstances and resettlement strains in host countries, such as Canada, infringe upon mental health and wellbeing. Prior scholarship concerning the psychological outcomes of refugees has been greatly criticized as being skewed in its rather narrow fixation on trauma undergone prior to and during the migratory process and consequently failing to account for the psychological impact of factors in post-migration ( $\mathrm{Li}$, Liddell, \& Nickerson, 2016). Refugee mental health is extremely complex, and coping with trauma is only one among a vast array of challenges that refugees face once they have finally arrived in a country of resettlement. A number of cross-cultural studies demonstrate that resettlement stressors can compound the psychological distress experienced by traumaaffected refugees, resulting in a heightened risk for mental illness (e.g. Miller \& Rasmussen, 2009). A conceptualization of refugee mental health therefore "requires the recognition of the role of both pre-migration trauma and post-migration stressors and that psychological distress can manifest in various ways depending on the biopsychosocial context" (Schweitzer et al., 2006, p. 180).

Pre-migration trauma, while critical to a refugee's experience, may not always been the most robust indicator of their mental health outcomes, particularly in the relative short-term after arrival. Respectively, Kim (2016) affirms that contextual factors in the resettlement environment 
are important determinants that contribute to the overall psychological well-being among refugees, beyond past traumatic experiences. Furthermore, research with resettled refugees that endeavors to locate the correlations between a multitude of structural and sociocultural determinants demonstrates that post-migration related stressors predict levels of psychiatric symptomatology better than pre-migration exposure to trauma (Miller \& Rasmussen, 2009; Steel, Silove, Bird, McGorry, \& Mohan, 1999; Porter \& Haslam, 2005). The various challenges refugees experience upon arrival and throughout resettlement are numerous, and particularly relevant factors include language barriers (Carswell, Blackburn, \& Barker, 2009; Kim, 2016), unemployment, underemployment, and living in poverty (Murray \& Skull, 2005), reception of the host country including discrimination and xenophobia (Hadley \& Patil, 2009), struggles in accessing healthcare services (Lamb \& Smith, 2002; Kim, 2016) acculturation related difficulties (Bhugra, 2004), changes in spousal roles and family dynamics (Deng \& Marlowe, 2013), inadequate social support and isolation, harbouring heightened concerns for the well-being of family remaining abroad including stress from limited contact and prolonged separation (Makwarimba et al., 2013), pressure to send monetary remittances (Schweitzer et al., 2006) as well as an identity conflict that is often intertwined with the label of being a refugee in a country of resettlement (Beiser \& Hou, 2006).

During resettlement, refugees endure a constellation of daily stressors that, although they may appear to be independent from, are instead likely to be exacerbated by the cascade of highly distressing and traumatic situations that they have experienced throughout the circumstances of pre-migration and migration. Silove (1999) contends that mental health is impacted by psychosocial factors in the post-migration environment either by protecting the individual from, or increasing the vulnerability to, past trauma and current stressors. This is affirmed in a study by 
Schweitzer et al. (2006) which tested the combined influence of pre-migration trauma and postmigration living difficulties in the determination of mental well-being in a study of Sudanese refugees resettled in Australia. Although this study was relatively small $(n=63)$, data revealed important findings that the traumatic experiences of refugees combined with psychological stress after arrival, and therefore that the effects of trauma and post-migration factors have differential impacts on different areas of functioning (Schweitzer et al., 2006). For example, while trauma appeared to directly predict symptoms of PTSD and somatization problems, difficulties in the post-migration context (e.g. language barriers, unemployment, discrimination, and acculturation difficulties) had a higher probability of being associated with increased depression and anxiety. Of particular note, results indicate that certain variables, such as social support and the presence of family members or a culturally similar community, are significant determinants that may protect individuals from vulnerability to psychological distress by mediating the effects of prior trauma and the ongoing stresses of resettlement. These findings are in line with previous research conducted by Beiser and Hyman (1997) which concluded that post-migration factors, inclusive of the emotional and instrumental support provided by marriage or an ethnic community as well as factors like success in obtaining employment, have the ability to mitigate mental health risks.

\subsection{The Relationship Between Time and Mental Health}

As demonstrated, a considerable body of literature exists on the effects of both past trauma and post-migration related stressors in the relative short-term; however, there is a dearth of longitudinal research that examines how mental health needs of refugees change over time in order to provide a more comprehensive understanding of the sequelae of pre- and post-migration experiences (Schweitzer et al., 2006). For example, it must be questioned as to why resettlement challenges are more telling of mental health symptomology in the short term, especially given 
that refugees have recently experienced such distressing events that caused them to flee their homeland. Additionally, further inquiry is needed to establish which determinants most influence refugee mental health in the long term to explore why "the psychological and emotional costs that are exacted on the refugee are enormous and lifelong" (Marsella et al., 1994, p. 6). This deficiency needs to be addressed in order to advocate the development of unique, socioculturally specific programs and services that expand the focus beyond the period of initial resettlement and are thereby accessed by refugees requiring continued psychological intervention.

While the long-term effects of trauma arising from pre- and post-migratory experiences on mental health symptomology is still debated, Ingleby (2004) articulates that traumatic situations and events in which refugees experience "are intimately connected with the rest of their lives" (p. 11). The after-effects of migration and resettlement on refugees are both wideranging in scope and powerful in their ability to result in complex psychological comorbidities that change over the course of their lives, drawing attention to the ways in which a multiplicity of challenges affects this population at all stages of their journey (Almqvist \& Broberg, 1999). This notion is substantiated by literature that has arisen out of one of the largest longitudinal studies of refugees entitled the Refugee Resettlement Project (RRP), which explored Southeast Asian refugees resettled in Western Canada between 1979 and 1981 over a 10-year period. Research by Beiser and Wickrama (2004) builds upon previous RRP investigations to examine the relationships between trauma, time, and mental health of refugees, specifically the phenomenon and mental health consequences of time splitting and temporal reintegration. Time splitting involves suppression of the past in order to dissociate it from the present and future, whereas temporal reintegration is conceptualized as a recall of the past and connecting it with 
both present and future. These scholars present Thomas Cottle's (1967) concept of time perception which encompasses both time dominance, "the relative importance attributed to past, present, and future", and time relatedness, "the interconnectedness among the three temporal spheres" (Beiser \& Wickrama, 2004, p. 900). Temporal reintegration for refugees can thus be thought of as the result of an increase in a dominance of the past, or nostalgia, and an increase in time relatedness in which their conceptualizations of present and future become reconnected to their past trauma, likely resulting in mental health issues as they realize what they have lost and left behind during the refugee process. The results of this study highlight that shortly after arrival in Canada, the time perspective of these refugees was characterized by dominance of the present and future and a time split from the past, including both isolated traumatic incidents and large blocks of personal history. Time splitting can therefore be used as a coping mechanism to protect psychological well-being in the immediate and mid-term aftermath of catastrophic and traumatic events. Accordingly, refugees deemed the present resettlement challenges, and their future place in Canadian society, to be prioritized upon early arrival over addressing past trauma.

Intrinsically, the longer time spent in Canada after resettlement, the more likely individuals were to reconnect the past with the future, which was shown to precede comorbid symptoms of depression, PTSD, and anxiety (Beiser \& Wickrama, 2004).

Two important conclusions can be draw from this research. First, in the immediate and mid-term aftermath of trauma, suppression of the past and its connection to present and future can be an effective adaptive strategy for coping with stressors, but trauma and other past experiences are highly likely to resurface at some point later on in resettlement. Secondly, Beiser and Wickrama (2004) highlight the pervasive power of a disturbing past, noting that "health and social service providers should be aware that depression-inducing recapture of the past can take 
place years and even decades after refugees arrive in a resettlement country" (p. 907). Premigration traumas do not suddenly become irrelevant, but instead Kim (2016) remarks it is logical that resettled refugees direct their focus towards prioritizing their immediate survival needs which dictate the quality of their present lives as opposed to those of mental health. Psychological need thereby influences an individual's perception of the past, present, and future, which suggests that interventions based on a trauma-centred premise could potentially be detrimental to long term mental health outcomes (Beiser \& Wickrama, 2004).

In a similar study using data from the RRP, Beiser and Hyman (1997) present the stress process theory and critical mass theory to explain mental health outcomes in refugees upon resettlement. Whereby the stress process theory proposes that events or experiences that overwhelm individuals (e.g. stressors) create mental health risk, critical mass theory claims that social resources provide a buffering effect against these stressors and help protect mental health. In confirmation of their hypotheses, findings from this research revealed that time splitting to avoid the past upon resettlement mitigates against depression in the short term, allowing individuals to devote their coping resources to stressors most salient upon arrival to Canada. However, the researchers further conclude that "when safety and predictability are restored, [refugees] will revert to time binding, and the past will re-emerge into consciousness" (Beiser \& Hyman, 1997, p. 1998), which is associated with greater risk of depression and other symptomologies of mental illness.

Although longitudinal studies of refugees that explore the relationship between mental health over time do exist, these analyses are rare. One such study of Cambodian refugees in the United States sought to assess the population prevalence of psychiatric disorders more than two decades after resettlement (Marshall, Schell, Elliot, Bertold, \& Chun, 2005). Results from this 
study indicate that despite the fact that an average of 25 years had elapsed since arrival, participants continued to reveal high rates of pronounced symptomologies, such as PTSD (62\%) and depression (51\%). While their analysis solely focused on mental health decades after resettlement rather than at different time periods, this data highlights that refugees may have substantial need for mental health services even years removed from past tribulations (Marshall et al., 2005). This finding supports the argument for initially prioritizing stressors that are rooted in the everyday resettlement context over prior trauma that is likely to be experienced as temporally distant (Miller \& Rasmussen, 2010) and the possibility for symptoms associated with the experiences of prior trauma to re-emerge when resettlement challenges have lessened (Gonsalves, 1992). In other words, refugees may not feel that dealing with trauma is a priority during resettlement in which the strain of post-migration factors are of more salience as they directly impact everyday survival. However, the multiple threats that are both concurrent with the initial trauma and for prolonged periods thereafter may never quite disappear, even after many years (Gonsalves, 1992). Past trauma that is often embedded within the process of forced displacement is likely to be compounded by multiple systemic and social determinants, shaping both short and long term mental health outcomes for refugees (Kim, Chen, \& Spencer, 2012; Kim \& Kim, 2014).

\subsection{Perspectives on Mental Health Service Provision}

Literature on mental health interventions for refugee populations resettled in Western countries is complicated by an divide between two divergent orientations of interventions (Miller \& Rasmussen, 2009; Nickerson, Bryant, Silove \& Steel, 2011). A critique has occurred of mental healthcare for refugees over the last decade, in particular that the documented dose-response relationship service providers have a tendency to rely on is a primarily pathological model that 
roots notions of refugee mental health within Western concepts of grieving whereby the ultimate goal is the prevention of emotional distress (Savic, Chur-Hansen, Mahmood, \& Moore, 2016). As international armed conflicts and the resultant forced displacement of millions of people have grown tremendously over the past few decades, refugee mental health has come to be growing field of study.

Miller and Rasmussen (2009) claim that, due to the increased attention and resources that have been directed towards the mental health of refugees by governments, researchers, and service providers alike, a clash in perspectives has developed between advocates of two fundamentally different approaches to understanding and addressing the mental health needs of this community. For advocates of a trauma-focused approach, because the most critical factor affecting mental health of refugees is the direct exposure to traumatic experiences prior to migration, psychological symptoms are believed to be ameliorated once the trauma no longer physically exists (Miller \& Rasmussen, 2009). However, certain scholars have recently begun to express an opposing perspective deemed the psychosocial approach, claiming that these formulations are problematic in that they accentuate individual pathology and gloss over broader social questions, culturally specific bereavement and their relation to social and community processes, as well resiliency in the face of persistent stress (e.g. Murray, Davidson, \& Schweitzer, 2010). In addition, this exclusively psychiatric model places emphasis on mental health interventions that disregard the importance of pre- and post-migration experiences in the development of mental health disorders both independent from and in combination with trauma (Miller \& Rasmussen, 2009). Psychosocial advocates posit that a trauma-focused approach to service delivery perceives symptoms of distress individuals may exhibit during resettlement to be a direct result of pathogens that are regarded as implicit in the refugee experience. 
Resultantly, this perspective may simplify these symptoms as being encompassed within a diagnosis of PTSD, resolved in a rather straightforward manner with a combination of therapeutic interventions and the passage of time (Savic et al., 2016).

An emerging pool of researchers and mental health practitioners have also critiqued the conceptualisation of PTSD, specifically arguing that it is a Western construction that pigeonholes refugees within a biomedical categorization and portrays them as passive victims of psychological illness (Watters, 2001). The cluster of symptoms that characterize a diagnosis of PTSD portrays violence and other forms of trauma as a preceding event in which the aftermath is a disorder that must be dealt with upon resettlement. As a consequence, an immediate diagnosis of PTSD fails to include refugees' own perceptions and interpretations of distress and how trauma affects their lives in the short, medium, and long term following migration (Watters, 2001). Additionally, this approach is problem-oriented rather than solution-focussed as practicing professionals may frame psychological interventions in epistemology that is underpinned by Western conceptions rather than culturally appropriate for different communities (Kim \& Kim, 2014). An assumption that experiencing trauma and other disturbing pre-migration events automatically renders refugee populations vulnerable to adverse mental health outcomes highlights an ethical and practical dilemma whereby a refugee's experience is condensed within a 'clinically significant' framework that pushes story-telling upon arrival under the justification that it has a beneficial and therapeutic effect for the individual (Watters, 2001). For instance, Turner states that "telling the trauma story serves not just to assist in the processing of the emotions with which it is associated; it is also the first step in the construction of a new story, a new understanding of the past and a new hope for the future (Turner, 1992, p. 171, as cited in Watters, 2001, p. 1710). Watters (2001) argues that the problem with this approach to mental 
health service provision for refugees is that their voice largely goes unheard. Instead of asking the individual to articulate what they want and need from mental health or other settlement services, they are primarily directed toward exploring traumatic events from their past and thus their resiliency, along with other needs that are perceived to be more pressing, are disregarded. Hence, Miller and Rasmussen (2010) state that, for refugees that have newly arrived in a country of resettlement:

The relevance and impact of mental health or psychosocial interventions are likely to be considerably enhanced when they are seen as targeting those sources of stress that are most immediately and severely affecting people. A programmatic focus on healing the effects of previously experienced [trauma] is likely to have limited impact on individuals who are facing ongoing exposure to traumatic stress in their homes or communities. (p.14)

Additionally, literature on refugee mental health accords that women are more likely than men to experience psychological illness, specifically because they are more at risk than men of experiencing sexual abuse and other forms of violence which seriously threatens their mental health (Miller \& Rasmussen, 2010; Thomas, Nafees, \& Bhugra, 2004). Yet without an intersectional lens that takes into account gendered specific instances of trauma and other determinants during the differential processes of migration and resettlement, literature such as this risks marginalizing women as fragile and more in need of support than men, who may experience and express mental health issues differently. It is therefore of crucial important to resist homogenising refugees into a single pathologized identity of PTSD and abstain from "the tendency to conflate the terms of refugee and war victim into a generalized category of traumatized" (Eastmond, 1998, p. 179, as cited in Watters, 2001, p. 1710). George (2012) claims that there is an ongoing and urgent need for service models to be developed that deal with past trauma when individuals are ready, but which also take into account the major relevant contextual issues that act as stressors to affect the daily functioning of refugees. This includes 
incorporating non-Western therapeutic interventions compatible with the indigenous values and traditions of differing refugee communities (Lacroix \& Sabbah, 2011). This will enable a shift in mental health services in countries of resettlement from being 'user-led' as opposed to 'serviceled' (Watters, 2001), and may in part explain why main-stream mental health services available for refugees in Western countries such as Canada largely go underutilized.

As highlighted, empirical investigations often present their findings through an explanation of a unidirectional dose-response relationship between trauma and mental illness which claims that the more cumulative trauma that has been experienced by a refugee in premigration, the greater the probability of them developing psychiatric disability such as PTSD and depression. Research such as this centers around a biomedical model of health care that may not be comprehensible to non-Western refugee clients. These studies are problematic in that they often assume that mental illness presents itself directly upon arrival in the country of resettlement and the refugee is ready to confront and manage past traumas through psychological interventions. Simply put, research such as this concentrates on the suppression of recall in the immediate aftermath of traumatic and horrific experiences that refugees unequivocally have experienced, and on the role services that target mental health can play to bring these issues to the forefront and alleviate suffering as soon as possible (Beiser \& Wickrama, 2004). As such, an interactional model of mental health may be more appropriate as opposed to the tendency of health care providers who strive to quantify the extent of trauma and emotional distress quickly after migration (Watters, 2001).

Based on the above review of existing literature, this study will provide a unique account from the perspective of the service provider as to how the mental health trajectories of refugees are complex psychological processes that likely require distinct needs at different stages of what 
has been deemed their 'lifelong' resettlement process. A substantial amount of research concerning refugee mental health uses quantitative data from symptom screening instruments to explore the direct effects model between refugee trauma and the manifestation of later psychological impairment. There is thus a gap in scholarship concerning the complexities between pre-migration, migration, and post-migration factors from the viewpoint of the practitioner providing mental health services to this population. It is important to note that while quantitative research is useful to gauge which issues are most salient to refugees in terms of creating mental health programs, research is frequently conducted while refugees are housed in refugee camps or alternatively within a short time after resettlement in a host country (Marshall, Schell, Elliot, Berthold, Chun, 2005). Few longitudinal studies have been conducted that aim to examine the scope of refugees' mental health statuses over different periods in time. While a longitudinal design is beyond the scope of this research, it is the investigator's belief that this study will be incredibly valuable in providing knowledge that that not only acknowledges the tremendous resiliency of refugees in the face of devastating life events, but brings awareness to how the mental health of refugees changes throughout resettlement. 


\section{CHAPTER 3: THEORETICAL FRAMEWORKS}

This study incorporated an intersectional social determinants of health framework as its guiding approach in order to understand and analyse how various pre- and post-migration factors combine with different facets of identity to enable and constrain intersecting social determinants for the refugee population. This chapter will therefore provide a comprehensive overview of both the social determinants of health and intersectionality paradigms in order to provide theoretical context for this research and a lens through which the findings will be explored and interpreted.

\subsection{Social Determinants of Mental Health}

The social determinants of health model initially came to the forefront of research during the 1970s (Marmot \& Wilkinson, 2006). The Lalonde Report, published by the Canadian government in 1974, first identified factors such as environment, lifestyle, and health care organization as social determinants of health, pushing forwards the importance of research on the socio-economic conditions that structure the health of individuals, communities, and the population in its entirety (Raphael, 2009). Simply put, this framework proposes that the root causes of inequalities and discrepancies in human health status are not biomedical or biological predispositions but are instead primarily affected by the ways in which governments construct and implement social and economic policies, and consequently distribute their resources (Hayes \& Dunn, 1998).

Canada has since identified twelve social determinants of health for the general population, including but not limited to income and education, social support networks, employment and working conditions, and personal health practices (Ip, 2016). However, this framework does not make reference to the complex processes of migration and resettlement, explained to be important social determinants to include because the individualized migration 
trajectories of newcomer groups intersect with a variety of settlement and social determinants, including gender, age, and race, to affect health outcome (Meadows, Thurston, \& Melton, 2001). Iyer, Sen, and Östlin (2008) posit that different axes of social power relations, such as gender, socioeconomic position, and discrimination and racism have been gradually recognized as "interrelated, not as additive but as intersecting processes" (p.13). Despite this recognized acknowledgement, there is a startling lack of research on the association between intersections of diversity, migration, and health. Specifically, there is a gap in scholarship in regards to how a combination of these variables cause social determinants to be further unevenly distributed, thereby producing additional inequalities for certain groups (Hollander, 2013).

The case of refugee health presents a number of unique complexities that do not exist for other immigrant populations, and their outcomes need to be interpreted within a multifaceted nexus of both individual emotion and broader social and political forces. This is due to the proposition that refugees are lower on the social hierarchy when viewed comparatively to both Canadian-born individuals and other immigrant groups. Arguably, refugee populations are therefore inevitably more likely to experience less favourable economic, social, and environmental conditions throughout their lifetimes that results in accumulative, psychological stress, and yet they have less access to formal resources (Allen, Balfour, Bell \& Marmot, 2014). With that said, negative mental health outcomes for refugees may be buffered by a combination of individual, family, and community strengths (Mawani, 2014; Galea \& Steenland, 2011). This highlights the need for a balanced view that acknowledges the role of protective factors that act as assets to safeguard against a decline in mental health, as well a deficit perspective that does not diminish the unequal socioeconomic landscape of the host country (Morgan \& Ziglio, 2007). 
Given the specific focus of this research, a social determinants of mental health (SDMH) framework (Galea \& Steenland, 2011; Solar \& Irwin, 2007; Mawani, 2014) is particularly amenable to locate and highlight the root causes that underpin mental health outcomes due to determinants that exist between refugees and the Canadian population, as well as within subgroups of refugees (e.g. men and women, LGBTQ refugees, older and younger). The SDMH framework promotes mental health across all societies regardless of their backgrounds and argues for the need to attend to structural and social factors that cause "injustices that determine and contribute to health disparities" (Kim \& Kim, 2014, p. 65). As such, Mawani (2014) proposed a multi-level framework that situates determinants of mental health inequalities across a continuum from pre-migration to resettlement, incorporating macro, community, family, and individual levels of determinants.

Encompassed within the macro level are the economic, political, social, and physical environments of both refugees' home countries and the country of resettlement. This includes crucial mental health determinants such as prior expectations developed for the host country preceding migration, the current political environment in refugees' country of origin, institutional exclusion, and societal-level income distribution, particularly when there is a sizeable shift in economic inequality from pre- to post-migration (Mawani, 2014). Refugees are often nested within many different and sometimes overlapping communities wherein mental health is affected by the demographic, social, cultural, and psychological composition. Factors at the community level therefore consist of access and availability of formal and informal social supports, cultural perceptions and expression of mental health, and the physical environment in which refuges live (e.g. housing). At the family level, social determinants affecting mental health may look different for refugees from non-Western regions due to the value placed on a 
collectivist rather than an individualistic outlook. Migration and resettlement can accordingly result in a drastic change in family dynamics, such as shifts in gender roles, intergenerational tensions, as well as severed nuclear and extended family supports due to separation and loss. Finally, individual level determinants make reference to migration status (e.g. refugee claimants and non-status individuals), contextual factors such as gender and sexual orientation, socioeconomic status, and discrimination (Mawani, 2014). Given the complexity of factors that are capable of intersecting and reinforcing one another to affect refugee mental health, application of the multilevel SDMH model is useful for the purposes of exposing unequal social determinants in order to problematize health inequalities.

\subsection{Intersectionality}

In light of the above, the construct of intersectionality is a valuable framework that can be used in conjunction with SDMH to more comprehensively understand the inequalities and disparities in mental health outcomes for refugees not only upon initial arrival, but also throughout their lifetimes. Intersectionality was first introduced as a concept in the work of such scholars as Kimberlé Crenshaw (1989) and bell hooks (1990), who sought to investigate and explain the marginalized experiences of racial minority women in relation to oppression and discrimination (Hallett, 2010). It exists as an important paradigm in various areas of the social sciences for the reason that an intersectional lens helps clarify and portray the multiple axes of social identity, such as race, gender, culture, and sexuality, that intersect to continuously impact individual lived experiences in a non-linear fashion (Ip, 2016).

Guruge and Khanlou (2004) propose a theoretical stance based upon the emergent concept of 'intersectionalities of influence' in order to create space for a novel exploration of the dynamic interplay of multiple sources of influence on the mental health and well-being of 
immigrants and refugees. This analytic apparatus posits that the individual health experiences of marginalized groups need to be conceptualized within the complex issues that arise at the intersection of identities that shifts away from single causal factors of illness to an exploration of how systemic and structural barriers are embedded within, and ultimately shape, these experiences (Guruge \& Khanlou, 2004). According to Langer (1990), the true and profound experiences of refugees cannot be fully understood without having a "clear sense of refugee trauma as having its origins in the intersection of history, social structure, and biography - an intersection that does not cease when refugees leave their homeland" (p. 69). Trauma does not exist in isolation from other inequalities for refugees, but instead must be interpreted through the context of power relations entrenched within the multitude of their marginalized social identities. An intersectional paradigm thus highlights the necessity of taking into consideration the other non-refugee identities that existed prior to migration in tandem with the erosion of those identities that have been greatly affected by trauma as well as by the preassigned refugee label and resultant inferior status in the post-migration context (Bartolomei, Pittaway, \& Pittaway, 2003).

Therefore, a perspective of intersectionality is complementary to the social determinants of health by facilitating critical inquiry of how "production, reproduction, and presentation of knowledge within a particular ideological foundation can not only perpetuate existing power relations, inequality, and vulnerability, but also result in further marginalization" of refugee communities (Guruge \& Khanlou, 2004, p. 35). These two approaches have guided the development of this major research project and the study's findings through an integrated perspective that recognizes the dynamic interaction between identity intersections and the operation of social determinants at multiple levels that directly affects mental health outcomes. 


\section{CHAPTER 4: METHODOLOGY}

\subsection{Research Design}

This study was guided by a qualitative approach referred to as qualitative description. A qualitative design facilitates in the production of "a complete and valued end-product in itself, rather than as an 'entry point' into other qualitative studies"' (Sandelowski, 2000, p. 335) and enables the advancement of knowledge based upon individual experiences (Thorne, ReimerKirkham, \& O'Flynn-Magee, 2004). Qualitative description does not stem from any specific discipline and therefore can have elements of other methods (Sandelowski, 2000). Qualitative description is interpreted "through (human) perceptions" (Wolcott, 1994, p. 13), and involves a comprehensive presentation of facts in the "everyday terms of those events" (Sandelowski, 2000, p. 336) that are not described in a way to fit the events into a system or predetermined hypothesis.

This method uses naturalistic inquiry and consequently reveals data that focus on the process, explore the micro level, and uncover vivid and rich knowledge through the unique account of participants' stories (Thorne et al., 2004). Qualitative description is particularly amenable to the research focus of this MRP because, to the best of my knowledge, this is the first Canadian study to provide insight into this particular topic from the perspective of the mental healthcare professionals who work with refugee clients. As such, it was important to obtain detailed information and stay close both to the data and to the surface of words and events. In addition, this research method allows the researcher to obtain "straight and largely unadorned answers to questions of special relevance to practitioners and policy makers" (Sandelowski, 2000, p. 337) as well as descriptive and interpretive validity (Maxwell, 1992, as cited in Sandelowski, 2000). 


\subsection{Sampling Technique}

This study incorporated purposeful and snowball sampling techniques in order to recruit participants from across the mental health care spectrum that work directly with refugees in the Greater Toronto Area. According to Creswell, Hanson, Plano Clark, and Morales (2007), purposeful sampling is a technique of non-probability that allows for a series of strategic choices in regards to the study participants, taking into consideration the specific inclusion criteria. By doing so, "the researcher intentionally selects individuals and sites to learn or understand the central phenomenon" (Creswell et al., 2007, p. 204). The eligibility requirements for participants were: currently employed service providers and had direct interaction with refugees for the purposes of providing mental health care and had work experience in a similar capacity for a minimum of three years. The latter criterion was based upon the desire to examine how the refugee population's mental health needs change with the passage of time. The decision to interview individuals involved in mental health service delivery as opposed to refugees themselves was due to in part to my positionality as a researcher combined with a paucity of scholarship that includes the voices of those who provide services to the refugee population. First, my positionality includes limitations in additional language ability and having 'outsider' status, and as such I did not believe I would be able to gain an accurate and comprehensive understanding of refugees' mental health care outcomes and needs from their own viewpoint. Finally, the choice to focus on the perspectives of mental health professionals filled a desire to address a gap in research by collecting information from and giving a unique voice to service providers rather than service users. 


\subsection{Ethical Considerations}

As the research design involved interaction with people, this study had to be approved under the merits of the Tri-Council Policy Statement: Ethical Conduct for Research Involving Human Beings (TCPS) by the Research Ethics Board at Ryerson University. The two central ethical considerations were: consent that is free, informed, and ongoing, and confidentiality.

In depth interviewing, gaining the participant's trust and understanding their concerns is crucial. Informed consent refers to "the knowing consent of individuals to participate as an exercise of their choice, free from any element of fraud, deceit, duress, or similar unfair inducement or manipulation" (Berg, 2001, p. 56). Before the commencement of each interview, the investigator provided all participants with a written consent agreement which they were asked to read and sign. As well, the investigator verbally reiterated the key points in the consent form, clearly stating the participant's right to stop the interview at any time, to skip or decline answering any specific questions, and to ask for clarification at any point. The consent form also provided information regarding the purpose of the study, what participation entails, the nature of voluntary participation, the right to withdraw at any point without giving reasons or affecting benefits/compensation, and the right to confidentiality of their information. In particular, the consent agreement highlighted that all identifying information of the participants, such as their identity and the agency or organization they are employed at, would be kept strictly confidential. Each interview was audio-recorded with consent from the participants to be transcribed verbatim later on in order to minimize biases and increase accuracy of the data collected. All participant involvement was voluntary and a small honorarium of thirty dollars $(\$ 30)$ was provided to cover such costs as transportation and time for taking part in the interview. 
To ensure confidentiality, the following precautions were taken: all identifying information of the participants (e.g. names and employers) was kept confidential and they were assigned unique participant identification numbers, the interview transcriptions were stored in a folder on a password-protected laptop, the interview recordings were erased once the research project was completed, and the transcriptions will be destroyed no later than 5 years from the time this research took place to allow for the data to be used for further academic purposes, such as conferences and journal publications.

\subsection{Recruitment Strategy and Participant Profile}

Once approval had been granted from the Research Ethics Board at Ryerson University, the investigator began researching Toronto-based agencies and organizations that offered and specifically catered their mental health services to refugees. To ensure voluntary participation, an email was devised and sent to gatekeepers of the identified agencies and organizations such as executive directors or representatives listed on the website as those generally responsible for responding to public inquires. This email contained details of the investigator, the study, as well as the eligibility requirements to participate, and requested their assistance in sending out an email to potential participants who fit the enclosed criteria. Once the appropriate gatekeeper granted permission, the investigator provided them with an email script to send out to employees who could then initiate first contact with the investigator via email if they wished to participate or request additional information about the study. To facilitate snowball sampling, participants were asked at the end of each interview if they were aware of any contacts they would feel comfortable referring to the investigator that fit the criteria for the study and may be interested in participating. 
In total, twelve agencies and organizations were contacted from which five participants agreed to take part in the study and were interviewed by the investigator. Interviews were conducted over the course of a three-week time period and ranged in length from 35 to 62 minutes, with an average of 45 minutes. At the beginning of each interview, participants were asked to self-identity their role or job title and years of experience working with refugees in their current role or a similar capacity for mental health care purposes (see Table 1). For the purposes of this study, the sample of service providers consisted of one clinical trauma counsellor, one psychologist, and three counsellor therapists.

Table 1: Participant demographics including identified job role, gender, and years of experience working with refugees for mental health care purposes.

\begin{tabular}{lll}
\multicolumn{1}{c}{ Participant Role } & Gender & Years of Experience \\
\hline Clinical Trauma Counsellor & Female & 3 Years \\
Psychologist & Female & 3 Years \\
Counsellor Therapist \#1 & Female & 9 Years \\
Counsellor Therapist \#2 & Female & 7 Years \\
Counsellor Therapist \#3 & Female & 5 Years \\
\hline
\end{tabular}

\subsection{Data Collection}

The method of data collection for this study incorporated in-depth, semi-structured, individual interviews which were audio-recorded upon consent from the participants. This approach helps to capture what is centrally important in their experiences and to uncover details as well as obtain a deeper understanding of the participants themselves, their views, feelings, and perceptions (Van den Hoonaard, 2012). Along similar lines, Patton (2002) claims that the 
primary purpose of qualitative interviewing is to provide a framework within which respondents can express their understanding about a certain topic in their own terms. The semi-structured interviewing method is useful because it provides the advantage of asking a fixed set of openended questions of all participants that draws upon themes of interest, but has a flexible structure in that it allows for elaboration, clarification, and unplanned questions to be asked (Berg, 2001). Berg (2001) states that "questions used in a semi-standardized interview can reflect an awareness that individuals understand the world in varying ways. Researchers thus approach the world from the participant's perspective... by using unscheduled probes... that arise from the interview process itself' (p. 70). This method leads to richer data because both the researcher and the participants are able to raise issues not directly addressed in the interview guide and the interview process thereby becomes iterative in that interviews conducted first may provide interesting information that can be incorporated into the interview guide for exploration with subsequent participants (Berg, 2001).

\subsection{Data Analysis}

Qualitative data analysis refers to "the process of making sense of the information you have collected and searching for what lies below the surface content... [it] is the fascinating process of making sense of what people have said, identifying patterns and understanding meanings" (Whittaker, 2009, p. 88-9). Data analysis involved thematic analysis, a method used for "identifying, analyzing, and reporting patterns (themes) within data" (Braun \& Clarke, 2006, p. 79). As explained by Vaismoradi, Turunen, and Bondas (2013), thematic analysis is a flexible research tool which enables narratives to be analytically examined by breaking the data into smaller units of content and identifying common threads that extend across interviews with the objective of attaining a condensed and broad description of the phenomena in question. This 
method of data analysis uses an open lens to focus on the relevance of patterns and facilitate contrasts and comparisons to arise between themes (Bryman, 2001).

The first step taken in the data analysis process consisted of immersion whereby the investigator prepared and organized the collected data by transcribing the audio-recordings of the participant interviews verbatim. Consistency was ensured by reading along with the transcript while listening to the interview and additionally reading through each interview several times in order to make notes based on initial thoughts. The next phase is reduction, in which a systematic approach to the data is developed. According to Forman and Damschroder (2007), three goals that the researcher should strive for during the reduction phase are to reduce the amount of raw data to only that which is most relevant to answer the research questions, break down the data into clear themes and thematic segments using codes, and reorganize the data into categories in a way that addresses the research questions. Codes therefore provide the classification system in order to analyze the data, allowing the researcher to thoughtfully and deliberately reorganize the data by themes and categories that are analytically meaningful and facilitate interpretation (Forman \& Damschroder, 2007). The investigator began with preliminary coding by reading through the transcripts line-by-line and colour coding passages by category that initially appeared to be potentially relevant to the research questions. After reading through all the transcripts, similar codes began to emerge from the participants' narratives and the individual categories of codes evolved into major themes that defined the concepts more broadly in terms of information. This enabled the developed themes and sub themes to be inductively analyzed and interpreted in a way that promotes a coherent and revised understanding in terms of the research questions, facilitating effective communication of what the data means (Forman \& Damschroder, 2007). 


\section{CHAPTER 5: FINDINGS}

The following chapter incorporates findings extracted from the analysis of the collected data. Selected quotations taken from the interviews are presented based upon key themes identified in relation to the research questions, as well as additional themes that arose during the qualitative process.

\subsection{Client Demographics}

In order to gain an accurate and comprehensive picture of the refugee population they work with, participants were initially asked about the demographic profile of their refugee clients. According to all service providers, their clients come from a wide range of countries, with the most commonly mentioned being: African countries; the Middle East, such as Afghanistan, Iraq, and Syria; Caribbean countries and; Eastern European countries, such as Hungary. In terms of age, two participants worked with children as young as three years old, but in general, participants stated that they primarily work with adults, ranging from the ages of 25 to 60 . The majority of participants described their refugee client base as predominantly female and, while one service provider conversely noted that the majority of the refugees she works with are men, the reasons for this will be explained in a later section. Finally, there was a consensus that the family structures of the refugees they work with greatly varies. With that said however, participants commented that both Government-Assisted and Privately-Sponsored refugees are more likely to arrive with their immediate family members because they have been selected from abroad, whereas refugee claimants must go through the more difficult process of escaping their country of origin and, therefore, family members are usually separated. 


\subsection{Resettlement Stressors Most Impacting Mental Health}

Across interviews, the resettlement environment was expressed to be extremely significant for refugee mental health. While it must be recognized that a multitude of stressors exist for refugees once they arrive in the country of resettlement, this section consists of postmigratory adversaries that participants perceived to have the greatest contribution to psychological distress.

\section{Language}

All service providers agreed that the most impactful resettlement stressor on refugee mental health is language proficiency. It underlies the ability to deal with all other aspects of resettlement following arrival in Canada, and as a result "there's a huge connection between language acquisition and mental health because it impacts your ability just to get what you need for yourself on a very basic but very profound level" (Counsellor Therapist \#3). Compared to refugees who come from English speaking countries or have studied it as an additional language, those who face language barriers encounter more struggles and therefore more stress, exacerbating any pre-existing mental health symptoms and leading to psychological decline the longer this language barrier exists.

To be honest, many of my clients right now tell me that the biggest stressor is not having the language because it prevents them being able to access so many things... Because they really do want to settle, they want to get jobs, they just want to be living as much as they can, and without that it hinders them. I see depression a lot because not having the language just creates so many barriers to accessing employment, to accessing services, everything. (Psychologist)

Participants also expressed that not only does a gap in linguistic ability impede on

survival factors such as obtaining employment and housing, but it also eliminates refugees' sense of autonomy by forcing them to rely on others for basic needs in which they have always been independent in their home country. 
I think it's one of the first barriers that they encounter once they reach Canada. For example, when they have to find an apartment, it's hard to find an apartment and they need someone to go with them and having to find volunteers all the time to support them and provide them with transportation and all that definitely becomes a hurdle. Which takes a toll on mental health because they're constantly stressed, constantly anxious... From the simplest things like being able to ask "how can I get here?" and "which bus should I take?" it becomes a lot more difficult. (Clinical Trauma Counsellor)

The importance of the ability to communicate in English was addressed by all

participants and, while interpreters do exist, most accentuated the large amount of stress that refugees face by depending on interpreters in order to access services and navigate systems, or else facing institutional intimidation by way of difficulties in communication. One participant who currently only works with the recently arrived Syrian refugee population recounted:

Many of the Syrians that have come to Canada have physical illnesses and so they were constantly gong to doctor's appointments and what not, which became an additional stress that they couldn't navigate the healthcare system on their own and they needed constant support with that. Getting interpreters, those are hard to come by sometimes. They just deal with a lot of additional stress, and that contributes to their mental health. (Clinical Trauma Counsellor)

\section{Social Isolation and Discrimination}

Lack of social support and resulting isolation was emphasized as a major contributor to mental illness in all refugees and that, "If that is addressed than there's so many other factors that automatically get addressed within that" (Counsellor Therapist \#2). While some groups of refugees may arrive as entire families, such as those who have been assisted by the government or sponsored privately by organizations or individual groups, refugee claimants have often had their family ties severed during migration, with members still remaining in their country of origin. Those who have been brought to Canada for humanitarian reasons or sponsored not only have family support, but also have additional resources waiting to assist them with resettlement and providing direction in terms of accessing supports. For example, several participants noted 
that the agencies they work at provide a 'first response' program for Government-Assisted refugees whereby "within a week of a refugee arriving to Canada, they would then have access to medical care, mental health care, and settlement assistance” (Counsellor Therapist \#2).

However, refugee claimants feel the loss of those support systems more profoundly because they likely arrive alone, which hinders their efforts to rebuild social networks and furthermore does not provide opportunities to find out about relevant services and formal supports. Migration trajectory and status is therefore interconnected with both access to services and the social inclusion that settlement supports provide for newcomers.

Moreover, many refugee claimants leave their families behind with the hopes that once they arrive in Canada and are granted status, they will be able to sponsor their families. As such, a large amount of individuals seeking asylum have left children, spouses, and other family members behind in countries where there is ongoing conflict and difficulties in transnational communication.

Family reunification is a huge issue in terms of affecting mental health... First of all, separation from systems of support is incredibly detrimental to mental health, but secondly people are often tremendously worried about family back home. Worried for their safety, they often feel a lot of survivor's guilt, they're worried about sending portions of their social assistance cheque back home, and just feeling a lot of responsibility and like they should be able to help more but still aren't established at all themselves here. That in itself I would consider its own form of trauma... I can't even explain what that does and what kind of impact that has on their mental health in terms of both the separation and the anxiety. (Counsellor Therapist \#3)

Service providers additionally stressed the role that shared experience plays in combating social exclusion, and therefore improving mental health. Although the process of forced migration is fraught with hardships and often horrific events, refugees may find ways to share their burdens with each other and, "by doing this, they create their own communities by standing together and just supporting one another" (Clinical Trauma Counsellor). One participant voiced 
the concern that she has clients who attend one-one-one counselling to deal with mental health issues but once they leave her office are extremely isolated in their personal lives, holding them back from making any further progress in treatment. Moreover, it was noted that even if refugees are nested within particular ethnic or religious communities, confiding in informal social networks is not common unless others are in similar situations and experiencing equivalent struggles. This counsellor therapist elaborated that her role is to subsequently transition that client from individual into group programs to intercept their isolation, thereby addressing the interlocking anxiety and depression that stems from feelings of loneliness and exclusion.

I have clients so often where they get to a position where they feel confident enough to take the steps they need with respect to doing things to gain employment and doing things for themselves, but then they feel like they don't want to go to a certain centre or place by themselves because it makes them nervous or uncomfortable. So then it's important for them to have that social inclusion where they can say "well a few of us can all go together" and there's that shared experience... I can't really articulate it, but knowing someone feels what you feel and understands makes all the difference. (Counsellor Therapist \#2)

An important factor that often has a role in producing social isolation, and therefore further exacerbates other resettlement stressors, is the discrimination that refugees experience in everyday society. Not only is prejudice and discrimination significantly related to increased psychological distress and lowered self-esteem at the personal level, but can also lead to worse labour market outcomes and lower socioeconomic resources. One participant explained that refugees are given a social insurance number which serves to differentiate them from other categories of migrants and permanent residents, and therefore employers may recognize their status and take advantage of their vulnerable and often desperate position. It was also voiced that poor treatment is frequently unexpected and comes as a shock to refugees who did not expect to face discrimination in Canada or have never experienced it before. 
I've also had a lot of clients talk about the racism and discrimination that they've experienced once they come to Canada, especially if they're coming from a country where their race is the majority or pretty much everybody in their community is of one race. Some people will talk about how they experience racism for the first time once they're here which becomes a new but huge stressor for them. (Clinical Counsellor \#3)

Likewise, while Canada has an international reputation as being open and a type of 'safe haven' for the LGBTQ community, it was revealed that many LGBTQ refugees have been disappointed with the amount of discrimination that they receive here. Participants noted that this group of refugees are particularly vulnerable given the extreme experiences of discrimination and oppression that they have faced in their home countries which was the root cause of their forced journey. Therefore, maltreatment upon arrival in Canada leads to more severe distress than other refugee communities.

I've had some LGBTQ community clients discuss with me how difficult it is once they come here. They really thought... Canada would be a lot more open, a lot more understanding, or there would be more access to supports and services here. But unfortunately they haven't experienced positive encounters, they say that they face a lot of discrimination even here in Canada. So a lot of them are upset with the treatment that they have received, and actually were hoping and expecting more. (Clinical Trauma Counsellor)

Several participants also commented on how, although the longer refugees are in the host country the more likely they are to feel integrated, "they always carry that label of being a refugee with them" (Counsellor Therapist \#2). As a consequence, refugees face discrimination not only within the large societal context, but also within their own smaller ethnic and religious communities as well.

\section{Employment}

Difficulties finding employment, underemployment in the form of deskilling, and lack of relevant education were brought forth as major resettlement stressors that negatively impacts refugees' socioeconomic status and subsequently their sense of self-worth and mental well- 
being. Participants expressed that many of their refugee clients knew that they would experience difficulties in gaining meaningful employment but are simultaneously surprised and disheartened that the challenges they face in trying to provide for themselves and for their families are so excessive. Particularly in the years immediately after resettlement, refugees did not expect to face the sheer amount of barriers to making a living, resulting in financial insecurity, and symptoms of depression. This struggle can be captured in the following:

Often times people are in employment situations that are way below their skill set, their knowledge set and their general expertise, and below jobs that they've left behind in their home country. So then they're earning money in significantly lower brackets than they probably should be and that contrast, having that life change, is also traumatic in its own way. They become more depressed, anxious, frustrated, irritable, angry. Often this can lead to them questioning if coming here was the right decision. Thinking that yes, they were running for their lives but at least there was a base level of stability and financial resources that they had access to versus coming here and feeling safer physically but having that difference in socioeconomic status... creates feelings of instability and ultimately not feeling safe in a different way. (Counsellor Therapist \#2)

Participants noted not only the struggles with socioeconomic aspects and unfamiliarity with labour market expectations that refugees experience, but also how gendered aspects of employment affect mental health. One participant observed that in many non-Western countries, traditional gender roles within family systems are valued, going on to elaborate that the majority of her female clients are homemakers because they lack education and work experience. Congruent with this sentiment, another participant further reflected that, when families are separated, "women might not have had any type of formal employment experience back home but come here and have to be the sole provider for their children or for themselves" (Counsellor Therapist \#3). As such, these women face not only the frustrations in obtaining a job but compounded anxiety due to finding themselves suddenly displaced in a role that may not traditionally belong to women in their home country and therefore have no prior experience 
navigating. However, for refugee families, economic provisioning is often principally considered a central male role. This in turn places an additional burden on the shoulders of refugee men who struggle under the stresses of learning the host country's language more quickly, advancing their education, and navigating the labour market in terms of credential recognition, lack of Canadian experience, and employer discrimination.

If they've come as a family, men often carry that burden of being the breadwinner which is made even more difficult when they realize their qualifications don't transfer or they need more education or they end up in a job in a much lower status than they were back in their home country. That majorly impacts mental health, not just the stress of supporting a family bur also the shame of being in a type of job where they're in a much lower socioeconomic bracket, often labour and jobs like that. (Counsellor Therapist \#3)

Men feel like they have a duty to be the provider and so there's that burden that's almost completely on their shoulders of finding work that will provide for their families so that they can afford a home and food and won't be on welfare or living in poverty. That becomes such a large stressor on top of finding a job in itself, that burden and that pressure of knowing that they may only have one income... and it's coming solely from them and so that's their responsibility. (Counsellor Therapist \#2)

\section{Waiting for Status}

The effects of acquiring status and the refugee determination process was not a topic that was to be explored as a major theme in the initial conceptualization of the research. However, it was one that was brought up by all but one participant and was therefore identified as an important factor that is necessary to consider in order to gain a comprehensive understanding of refugee mental health. For example, several participants described the process of obtaining refugee status as the biggest resettlement stressor that affects refugee mental health for claimants due to its impact on multiple levels of resettlement:

Waiting for status is this all-consuming, all-pervasive thing... If you think of trauma as an experience of profound unsafety, and then the impacts of trauma as being really connected to ongoing feelings of not being safe, not having assured status in Canada means that you're psychologically dealing with the constant threat of having to return 
to the extreme abuse that you faced. That becomes a huge barrier to healing from past trauma if you don't feel confident or assured that you won't have to return to the types of trauma you left behind. (Counsellor Therapist \#3)

Additionally, participants discussed specific clients during the interview in order to paint a picture of how filing a refugee claim and waiting for a hearing, and ultimately for refugee status, infringes upon mental health. This is of importance to highlight because, as mentioned, this was a recurrent sub theme across interviews but is an understudied topic within the Canadian context in which very little research has been dedicated. The discussion on non-status individuals prompted service providers to note that waiting for status significantly affects mental health not only because of the constant anxiety they experience while living in a space of precariousness, but also because not having status disallows individuals from accessing education, gaining stable and legal employment, as well as finding out about available supports through social networks and subsequently connecting with necessary services. The process of obtaining status therefore has a detrimental and continuous impact on mental health because, as a participant explained, the individual is constantly preoccupied with the anxiety and uncertainty of what will happen to them, wondering "Will they be killed, tortured, imprisoned? Sometimes they are not able to function in their daily life due to being so anxious and depressed about their delayed or cancelled hearings" (Psychologist).

All participants, except one who works within an organization designated as a first contact for resettled Syrian refugees, noted that the majority of the clients they see are those who have been granted refugee status once in Canada or refugee claimants. There was an agreement that one of the foremost reasons why Government-Assisted and Privately-Sponsored refugees are the minority of clients seeking mental health services is due to the fact that they arrive in Canada with the assurance of permanent residency status. Further emphasizing the toll that the process of 
obtaining refugee status takes on mental health, an interview with one participant who regularly writes psychological reports for refugee hearings revealed how navigating the bureaucratic hurdles of the current system re-traumatizes clients during this time because, "they continue to have interviews and meetings with people where they are being asked again and again about [their story]" (Psychologist). Another service provider similarly critiqued the system by explaining:

In this process it's like their trauma is framed as a type of currency that they have to trade for access to things that they need in order to survive, such as status, access to services, [and] inclusion... [Refugees] are faced with this already highly pressurized situation and then they have to share the horrible things they've been through to strangers in positions of power, with the constant threat hanging over their heads of not being believed which ultimately means returning to what you've worked so hard to escape. (Counsellor Therapist \#3)

The relationship between classifying trauma according to the Western merits of a

psychiatric model of mental health was criticized by participants as lacking cultural-sensitivity.

In terms the refugee determination process, striving to fit the diverse experiences of refugees within the confines of a PTSD diagnosis both ignores other aspects outside the scope of their pre-migration trauma as well as renders symptoms expressed after undergoing horrific events as psychological illnesses rather than normal reactions to trauma.

I think there's a big pressure to pathologize people in order to feel like the Immigration and Refugee Board will take their trauma seriously, but pathologizing refugees runs the risk of invisibilizing this sociopolitical issue by making it into an individual illness. To me, 'refugeeness' is a sociopolitical phenomenon not a medical phenomenon.... which I believe is perpetuated by systems of power that places value on that. So if the judge is going to take an individual more seriously if they've been formally diagnosed with PTSD, then the professionals writing these reports are going to be more inclined to give them that and look for that within their experience. (Counsellor Therapist \#3)

\section{Acculturation Challenges}

Finally, clashes between values in the refugees' countries of origin and Western ways of living was expressed to create additional stress for refugees on a variety of levels. One of the 
biggest adaptation challenges that arose from participant narratives is how many refuges come from societies that place a great amount of emphasis and value on family in a collectivist manner. However, they discover upon resettlement to Canada that the Western mode of individualism is extremely foreign to them, impacting social inclusion, access to resources, and additional stressors such as employment. As such, there is a dramatic shift in their sense of identity, social relations, and cultural practices that they once viewed as a source of comfort and strength, particularly for refugees who arrive alone and therefore lack their immediate family as sources of support. When speaking about acculturation factors that often result in mental health decline, one participant communicated:

The other big aspect that contributes to this is a lack of community and how individualized our society is, and that's something that greatly impacts them because many refugees come from collectivist cultures that are very family oriented, very social, and very outgoing. Here they notice that there isn't that kind of life, everybody keeps to themselves, most of the time people here don't really even know who their neighbours are, and so they feel quite isolated. That social aspect is something that they miss a lot. (Clinical Trauma Counsellor)

Further acculturation challenges articulated by service providers was the contrast between expectations that refugees have of life in Canada and the unanticipated reality once here. It was expressed that resettlement challenges are made more difficult as refugees are typically not aware of the extent of these hurdles beforehand and arrive with different conceptions and expectations. These preconceived notions about the receiving community play an important role in clients' psychological health due to the lack of mental preparation for what life would be like in Canadian society. Thus, their perhaps unrealistic expectations were met with unforeseen, extremely stressful and emotional difficulties. Providing one example, a participant discussed expectations around Canada's refugee determination system: 
They mostly expected to have the progressive road to obtaining status. A lot of them have an understanding that it's going to be a lot faster, a lot easier, and there's going to be a lot more support than it has turned out to be. A lot of people are not prepared for the often three-year process that it takes to go through the hearing and get status, and are also not prepared for the fact or informed that going through the process doesn't guarantee acceptance and that they might actually have to go back. (Counsellor Therapist \#2)

Participants regretted to note that their refugee clients are often mislead about the types of formal supports available, and were highly disappointed upon coming here with the lack of services offered. A possible explanation for this was elaborated upon by one participant who revealed:

Some of them expected that they wouldn't have to pay for housing and financially there would be more support for them... some of them just thought that life would be easier here [because they] expect that life in the West is just easier and that everything is always available. But then they found out that no, life is actually harder here. (Clinical Trauma Counsellor)

The intersections of identity and culture impact refugee mental health, and are therefore important factors to be considered within their lived experiences of acculturation. In term of gender, domestic abuse was identified as a factor that predominantly affects refugee women. While several service providers commented that this was something their female clients expressed fairly often, they were unable to conclusively say if the abuse had precipitated coming to Canada and went unreported, or if it occurred as a possible result of the stress of acculturation and resettlement. One participant affirmed that "the female role in certain countries is really of a second-class citizen. They are really dependent on the man, so there's that control and dominance which takes a toll on women's mental health" (Counsellor Therapist \#1). The internal discord that refugees face during resettlement, especially those from non-Western countries, was emphasized to arise from cultural conflict and be particularly difficult for women because they are typically expected to be caregivers as well as the bearers of culture. 
I think that for a lot of refugee women, the option to be independent in multiple ways... is very difficult for them. There's a lot of internal conflict that typically arises with respect to wanting to maintain and uphold certain aspects of their culture and their identity which may be attached to religion, or their ethnicity, or where they come from. But also realizing that they now have options that they feel they want to utilize given the fact that they're now living in a Canadian context. It becomes very difficult and a lot of women struggle with that and figuring out their boundaries and what to do. (Counsellor Therapist \#2)

Another participant explained implications for women suddenly finding themselves in a country that accepts and celebrates female power and independence:

There has been a lot of change when it comes to women refugees coming to Canada... because they've gained a newfound power, a lot of rights that, even though they are still in their religion... they're no longer immersed in the culture. And so this has caused a lot of chaos for some families, some clients, in their households as to how to navigate this, how to deal with this newfound power. They are having to redefine the roles in the family. (Clinical Trauma Counsellor)

As well, LGBTQ refugees were perceived as having a very unique and individual migration experience, while also being a vulnerable population that are affected by specific acculturation impediments. Systems of homophobic oppression in the countries from which they've fled clashes with that of Canadian society, and is a cause for refugees to feel a range of conflicting emotions because they have "received messaging for the majority of their lives that who they are is not okay, is wrong, is taboo, and is just generally all of these horrible things" (Counsellor Therapist \#3). Another participant elaborated:

I had a lot of clients who were initially very happy and relieved because they felt like "I've made it here and now I can be who I actually am" and all of that. But that feeling was immediately contrasted with feelings and thoughts like "Oh, but in order for me to have that, I had to give up absolutely everything that I was once connected to" and as a result they were also extremely depressed and felt very alone and also very guilty about the obligations and the roles and the expectations that they had to leave behind. Specific to that group of LGBTQ refugees, I found that there were a lot of people mentally struggling with a kind of two-sided thing where it's like "I finally feel really happy and safe, and I feel really guilty and horrible about that at the same time". And that combination is very much specific to that group versus the other groups of refugees with different identities. (Counsellor Therapist \#2) 
Finally, age of migration was expressed to be intimately connected with acculturation challenges. Several participants spoke about how a defining feature they have noticed in refugee children is there impressive resiliency and ability to adapt to a new way of life, whereas older refugees may face more stress when confronted with clashes in culture upon arrival to a Western country. Therefore, age of migration is an important factor to consider in refugee mental health whereby older refugees tend to struggle more with adaptation, language acquisition, and other resettlement aspects such as social inclusion.

Age definitely plays a role in terms of how mental illness and stress affects the person and their internal understanding of that. In some cases, if the client was older, that would mean more resistance to certain things once they came here because they felt a lot more attached to some of the beliefs and outlooks that they had carried with them for most of their life... [it] affects a person's ability and capacity to do more things for themselves sometimes as well. (Counsellor Therapist \#2)

\subsection{Barriers to Accessing Services}

Three systemic barriers were discussed in terms of barriers to accessing mental health care: stigma and lack of psychoeducation, expression of mental health, as well as funding and types of services offered.

\section{Stigma and Lack of Psychoeducation}

Stigma was heavily emphasized by all participants as a significant cultural constraint that prevents clients from seeking help because they wish to refrain from potential stigmatization by their family and communities. It was described that mental health issues in many refugee producing countries are hidden because it's perceived as the individual being 'crazy', and therefore these beliefs are carried with them when they migrate. A clinical trauma counsellor observed that, even when refugees recognize that they need psychological help and want to access services, stigma is so deeply embedded within their perceptions of mental health that it hinders their access to it. She noted that, "I've had clients ask me if we could meet somewhere 
private, because I do a lot of home based therapy, so they don't want to meet me even in their homes because other people will find out" (Clinical Trauma Counsellor). Service providers additionally concluded that often times refugees would rather suffer in silence than risk others knowing about their issues. According to one participant:

...they usually don't want to talk about mental health. It's something they don't want to be affiliated with, any kind of mental illness or any mental health issue, so they just choose not to discuss it...Actually something I see all the time is psychiatrist versus psychologist. Even if it's just for a psychological report [for their hearing], clients say "no, could you refer me to a psychologist and not a psychiatrist?", and I will ask them “okay, so why?". It's because they don't want people to know that they're seeing a psychiatrist. It's the stigma. If their friends or family know that they are seeing a psychiatrist, they would think they've gone crazy. (Clinical Counsellor \#1)

Though stigma has been extensively identified as a major theme in refugee communities generally, mental health services targeted towards refugees are often approached in a manner that does not acknowledge both the stigma that underlies mental health issues in a large number of non-Western countries alongside the subsequent lack of psycho-education offered. Making reference to a specific client who attended counselling for over six years, one participant spoke about how mental health services are a culturally alien process to refugees and how services promoted within a Western conception of mental health are either stigmatized or not understood.

If you ask this client, "for the past six years when you've been going to [name of agency], were you regularly seeking mental health services?" she would say "no, I don't think so". But if you asked her things like "were you going there and getting support? Were you seeing someone who was helping you find ways to cope with stress?" she would say yes. If it's framed in terms of mental health, that's not something that refugees really feel resonates with them... It's the way in which that same concept and that same issue is worded that really makes all the difference with respect to access of services, with respect to people being willing to use the available services, and just being open to the process... Even the way that I articulate my role and my purpose to my clients is very specific and done in a way that utilizes language and approaches that I feel are more relevant to them and to their needs as opposed to the technical definition of my job. If I say I'm a counsellor therapist, what does that mean to them? (Counsellor Therapist \#2) 
Gender differences were also acknowledged in terms of how appropriate mental health issues are thought of for men and women. Males typically face additional stigma due to the systemic way in which psychological distress is conceptualized in many non-Western countries.

For a man... it's not okay to feel those issues. It's weak if he feels down or other symptoms that make him appear to be not doing well so it's not okay to feel bad which really impacts their ability to get better because there's so much self-judgement going on about it, never mind telling other people. (Psychologist)

Throughout the interviews, several service providers noted that their male clients tend to be more tense and unwilling to open up about issues going on in their lives compared to their female counterparts. While one participant commented that the majority of her adult clients are men, it was clarified that women are much more likely to self-refer and access mental health services on their own because they are more aware of their mental state, whereas men access services only when their doctor notices psychological symptoms and set up the appointment for them. She went on to say, "I think overall it's very much a stigma issue... Conceptions of masculinity also play a large role in that which ties into and perpetuates stigma surrounding mental health and mental illness" (Counsellor Therapist \#2).

\section{Expression of Mental Health}

How psychological issues are expressed by the refugee population was also determined to be a systemic barrier faced by both refugees and service providers in providing mental health assistance. It was explained that the majority of refugees have not encountered services specifically to address mental health needs in their home countries. Consequently, when they arrive in Canada, refugees do not understand what mental health services are. Therefore, "refugees won't go out of their way if they don't have to in order to access mental health support" (Clinical Trauma Counsellor) and, "a lot of times they are more apprehensive or not really understanding why talking to someone else about what's going on with them could be 
important or could make a positive difference" (Counsellor Therapist \#2). Every participant made reference to a blurring of lines between physical and psychological symptoms, somatization, for their clients, which they proposed to arise due to a lack of psychoeducation intertwined with the stigma embedded in addressing mental health. For instance, one participant stated that being sensitive to cultural idioms of distress is critically important for those who provide services to refugee populations across the healthcare spectrum because,

They more so think that their [mental] illnesses are physical, and so they go see a doctor... they tend to have somatic symptoms and so they have constant doctor appointments but no real results. All the results come back and it's like "you're in good health", but it's not physical. It's definitely psychological. (Clinical Trauma Counsellor)

\section{Types of Services and Funding}

As previously mentioned, although the aggregated results demonstrated that the participants classified the majority of their clients as women for the reason being "almost 80 percent of refugees are unfortunately women and children" (Counsellor Therapist \#1), one participant described the way in which gender and culture of merges to become a barrier to accessing services for her male clients:

I think because of the Syrian population and their culture, my gender makes it difficult for men to access my services. Just because in Syrian culture and because of religion, men and women don't typically tend to mingle as much and because I'm female, men may not feel as comfortable opening up and discussing their issues as they would with a male counsellor... I know they definitely don't tend to access the mental health sector as much as women, but the ones that do are in dire need of these services. (Clinical Trauma Counsellor)

Despite this, it was further expressed that there are few mental health supports that specifically cater to men, especially Arabic speaking men. Within this same notion, the shortage of appropriate mental health services and resources that are both culturally-specific and catered exclusively for the refugee population was emphasized. Participants spoke about how services 
do exist, but that a lack of the 'right' services puts pressure on the resources that are culturallysensitive, ultimately impacting the mental health outcomes of their clients. According to one participant:

Just because of our caseload, sometimes unfortunately we are not able to see them as much as we want to, as much as they need to... Even for us, refugees come and they are on the waiting list for a while. When you are on the waiting list and you have a mental health issue going on, what happens? You don't get better, because you are on the waiting list. You live with it; you even get worse. (Counsellor Therapist \#1)

Discussion regarding a lack of types of services lead many participants to comment on funding, specifically remarking on how funding is embedding within the process of creating services for newcomer populations.

... services that are specifically designed and catered towards refugee mental health are really overused because there just aren't enough services like this. There's just not enough resources to put the amount of services that are needed for this population in place, a lot of it really comes down to funding. (Counsellor Therapist \#3)

As a result, a pervasive theme that arose from the data was that of a lack of funding from the government, which acts as a barrier for organizations being able to provide assistance to the necessary demographics and consequently for refugees to accessing services. Particularly when it comes to mental health, there was an agreement across interviews that, "There's a lot of services helping with and teaching about resettlement, things like computer skills... But in terms of mental health, I think there's definitely a lot more that could be done if there was funding for it" (Psychologist).

Given that they work in the settlement sector, participants were especially passionate and keen to discuss why funding is designated to particular newcomer services and supports above others, and the affect this has on multiple levels. Generally, the notion was brought forth that the way in which funding is distributed in a top-down manner to newcomer services is determined based upon those in power defining which groups are a priority and, as a result, "refugees just 
get lost in that conversation, their actual needs and the actual people are lost in that" (Counsellor

Therapist \#2). Similarly, another participant concluded that, "refugees are quite a marginalized

group and so often marginalized groups don't receive the same amount of visibility and therefore the resources that other communities do" (Counsellor Therapist \#3). While the desire for different settlement organizations to share resources was understood, participants raised concerns about how this may appear to be efficient but actually results in cookie-cutter services that leans towards a Western medical model of mental health as opposed to one that is client-centred and culturally appropriate. The struggle for funding therefore results in services that are not conducive to the actual needs of the clients they are trying to acquire funding for in order to assist. However, agencies take this approach due to the way that they understand funding to be allocated and the competition for monetary resources within the settlement sector.

It's driven very much by agencies and organizations aiming for their mental health teams to hit a certain quota and have a certain amount of people seen and using their services in order for them to get funding... But then the analysis that's being done isn't accounting for that bigger picture, it's just looking at "well this one person came in, utilized this service a few times and then left", and the stats just aren't reflecting the reality of what's happening behind that. The problem with that is that the funding is based on the stats, and that's a big issue because it appears as though people are being helped but they aren't. (Counsellor Therapist \#2)

The possibility of Legal Aid getting reduced or cut completely so refugee claimants

would have limited to no access to legal representation for their status determination hearing was also brought up within the sub theme of funding by a number of participants.

I think that it may have come out of a... perceived need, to filter out the group of people within the newcomer and refugee population that people feel are exploiting the system. And so their thought process is that the more free services that they remove, that's going to filter out those who are exploiting that system and that those who are legitimate will somehow find the funds and find the supports. It's a very flawed logic... I think it's highly problematic, it's very short-sighted, and it's of an old Conservative mindset that is no longer relevant to what's happening in the world today. (Counsellor Therapist \#2) 


\subsection{Resiliency}

The theme of resiliency and coping strategies refugees utilize in the face of psychological distress was a recurrent theme across interviews. All participants were eager to highlight the incredible resiliency of the refugee population and noted that, while the majority of their clients exhibited comorbid psychological issues such as Depressive Disorder, Anxiety Disorder, and PTSD, coping comes from an internal mechanism trigged by the need to survive and move forward with their lives despite the cascade of trauma refugees have often been through. Specifically, one counsellor therapist discussed how she was constantly impressed that many of her clients were even able to get out of bed each day, some which have seen their children or other family members and friends die in front of them and then have additionally faced extremely difficult circumstances during all stages of their migration process.

When situations for them are so dire and so severe, people's need to survive and come through that is what gets them through... when these people are faced with yet another stressor or trauma in what has probably been a long line of adverse situations, internally there's an instant default to compare past stressors or trauma and say to themselves, "this is going to be okay because I was able to see past this and I already survived" ... I think that is specific to groups of people who have gone through very high levels of trauma and have come through it, such as refugees. (Counsellor Therapist \#2)

Of interest, and contradictory to literature on this topic, one participant commented on the difference in resilience and resourcefulness between refugees who had previously lived in refugee camps and those who did not. In particular, it was highlighted that a certain hotel in Toronto had housed a large number of refugees while they were being resettled who had all resided in the same refugee camp in Jordan:

They're just extremely resilient, they found ways to navigate the system themselves, they seemed to adapt to life here a lot quicker. They also didn't need as much support as the ones who came from urban centers and were used to a particular lifestyle. A lot of them in urban places came from money and they had the supports in Syria, or even after when they were forced to move to Jordan or to Lebanon, so coming here was the 
first time they didn't really have anything and they had a lot more difficulty starting from zero. (Clinical Trauma Counsellor)

Interconnected with a lack of psychoeducation and differences in expression of symptoms, participants also explained basic therapeutic coping techniques they use with their clients. As one participant elaborated, "I just teach them to breathe sometimes. That's so basic and fundamental to calm down when they're anxious, and they've never heard of it before" (Psychologist).

There was unanimity among participants that religion and spirituality are interconnected with resiliency. Leaning on religion therefore becomes a coping strategy in and of itself by functioning as a means for refugees to deal with their psychological distress resulting from both pre- and post-migration circumstances. Spirituality and religion was explained to be grounded in cultural values, and also as a natural path to most easily develop a sense of community and social inclusion in an unfamiliar environment.

I think that in general a positive spirituality makes it easier to find a sense of meaning and hope... I think that refugees tend to lean on their religion and spirituality more because they've had so many other resources taken from them but faith is something that they can always carry with them wherever they go, whether they're in refugee camps or a new country trying to make a home. Religion and faith is also a way for them to find and build a source of community in the place that they settle, they can connect to community in their place of worship and it facilitates social connection in that way. (Counsellor Therapist \#3)

\subsection{Changes in Mental Health}

Even though all participants emphasized that not only is mental health for refugees a complex process affected by a multitude of influences and therefore very much individualized, themes related to how mental health for refugees can change over time are grouped into two subsections. The first covers the role of prior trauma as a contributor to mental health changes 
and longer-term outcomes. A second section describes how resettlement factors directly affect psychological fluctuations for refugees.

\section{Influence of Pre-Arrival}

Examination of the data in relation to factors that impact changes in mental health pinpointed circumstances that exist for refugees during pre-arrival, such as undergoing trauma in the country of origin or in transit. While the resettlement environment is extremely influential on mental well-being, this context must be interpreted with regards to pre-existing conditions and earlier exposure to different types of trauma that not all refugees experience to the same extent. For example, one participant noted that refugees may have pre-existing biological mental illnesses, such as schizophrenia, bipolar disorder, and borderline personality disorder. These issues intersect with potential trauma and resettlement stress, creating additional barriers and disadvantages upon arrival.

The role that experiencing what is often an intense accumulation of trauma is one that impacts an individual's psychological well-being on every level. However, despite many refugees experiencing severe trauma, resettlement remains a priority for them as opposed to the often debilitating mental health issues that affects daily functioning. It was indicated, both directly and indirectly through participants' narratives, that while trauma can be mitigated by decreased resettlement stressors and coping, it will always surface in some way and need to be addressed. A psychologist stated that:

I think people are also initially trying to focus on survival and resettlement ... Even if it's three years later, I still find that people typically have a lot of symptoms. But it often hasn't been addressed... The pain, the memories, the thoughts that come up are too painful. So they try to push them away so they don't have to deal with them, but then they surface and come up in different ways. (Psychologist) 
As a result, stressful resettlement hinders healing by exacerbating trauma, which perpetuates mental health symptoms through re-traumatization. Even if resettlement is less stressful for certain individuals, trauma still arises at some point, impacting the lives of many refugees years into their resettlement. To illustrate, a participant remarked:

...but these trauma symptoms can come up months, even years down the road still because unfortunately they don't disappear during the time when other things are going on, it's more just like it's been put on hold and the things that are happening can either help relieve some of that trauma by stabilizing or making it worse by causing more stress, more uncertainty, and overall more instability. (Clinical Counsellor \#2)

Of interest, two participants brought up how trauma affects specific aspects of resettlement, which has a domino effect on other factors that ultimately hinders successful settlement and integration. Trauma therefore triggers a stress response which exacerbates and perpetuates symptoms such as recurrent and intrusive recollections, flashbacks, and nightmares.

[Trauma] has a huge impact on things like learning because it affects the ability to remember, it disables processes like memory retention, concentration, and focus. It can be very difficult for people sit in a classroom and stay focussed and try to remember things... So just in general, language acquisition can be much more difficult for refugees compared to other newcomer groups... If you can't learn the language in the way that the services are offered because of trauma, then you stop going to classes. And then upgrading your education becomes nearly impossible, so you can only find work in a job that's way below your skill level. From there you get depressed and anxious about money and the more depressed and anxious you get, the more your trauma symptoms come to the surface, for instance the person may start to have nightmares more frequently. And the more that happens, the more every single part of settling in a new country and trying to find your way becomes this massive barrier. (Counsellor Therapist \#3)

Resettlement stressors were additionally portrayed as a hindrance to confronting past trauma, thereby serving as a barrier to healing from the experiences that refugees have faced.

This is due to the fact that, "Refugees don't want to immediately address their past trauma. They want to feel more stable and secure... Until those aspects of their lives have more definition, the anxiety around that is what we are often working with first and working through" (Clinical 
Counsellor \#2). For instance, one participant elaborated on a long-standing client who fled to

Canada seeking asylum on the basis of her sexuality being criminalized in her home country. This client is referred to as a 'legacy claim', meaning she arrived before the overhaul of the refugee determination system in December of 2012 and therefore, due to an extensive backlog, many of these claims have not yet been assessed by the Immigration and Refugee Board (IRB).

... so she hasn't been able to go back to school, she's had to renew her work permit every year, and she lives in that constant fear of not knowing if she'll be allowed to stay... there still remains this massive barrier of status that is holding her back from resettlement and also from confronting her past trauma and the substantial amount of loss that she has faced in order to come here. (Counsellor Therapist \#3)

As a result of waiting many years for her status determination, this particular client finds herself unable to participate in everyday society, preventing her from being able to fully resettle which is tied into her past trauma that she endured on route to Canada. In this way, trauma either may not present itself until resettlement becomes more stable, if and when it does, or refugees may be experiencing the effects of trauma in comorbid psychological symptomologies (e.g. PTSD) but are not ready to confront and address it until post-migration stressors at least partially diminish.

To assess how mental health changes over time, participants were asked to recall and discuss actual clients they have seen over a number of years. A clinical trauma counsellor described a woman that had arrived in Toronto four years ago after witnessing the beheading of her husband and then subsequently being kidnapped along with her two children, imprisoned, and frequently raped and tortured. The participant elaborated that her client is recurrently triggered, resulting in dissociations where, "She will be doing something and all of a sudden she's not present anymore, she will be on the floor kicking and screaming and reliving a memory" (Clinical Trauma Counsellor). Although her basic settlement needs have been met, symptoms of PTSD have caused this to struggle with severe social anxiety in public, and she is 
thus unable to attend educational classes and obtain employment. Her trauma has consequently

resulted in social isolation and depression which only further exacerbates her symptoms.

However, despite her awareness of her compromised functioning,

She doesn't really tend to talk about her trauma, just because I think she's still not ready, it's just too much for her... She goes through cycles where she's highly triggered and other cycles where she's not and she's okay... Maybe she will open up more in the future, but it's been four years and she's still unable to talk about it. It's very difficult for her. (Clinical Trauma Counsellor)

\section{Influence of Post-Arrival}

A salient theme across interviews in regards to how mental health changes was the extent to which the resettlement environment in Canada factors into psychological outcomes for refugees. While many refugees have experienced a migration trajectory that has involved at least some degree of trauma, participants underscored the fact that the stresses of resettlement in and of themselves have the ability to shape mental health. As one participant summarized:

... a positive change is really dependent upon how quickly they are able to resettle and have stability within the entirety of the resettlement process... If those settlement supports aren't there or aren't approached in a way that is effective for the client, changes over time can get worse because of the stress they experience... If that happens, they may live a good portion of their lives in a state of instability. (Counsellor Therapist \#2)

Furthermore, there tends to be a strong mutually reinforcing relationship between

psychological issues and the stressors of resettlement, expressed across all interviews. Often, refugees arrive with low mood, limited energy, and a lack of social supports, affecting their capacity to navigate the various resettlement challenges that they face and thereby sustaining depression, anxiety, or other mental health issues. A counsellor therapist explained:

It's very vicious cycle because they come here already in a certain distressed psychological state, and then initial resettlement is a very stressful... But the more stressed and anxious they become, the more difficult it becomes to navigate through the hurdles and stresses of resettlement, which often makes them more depressed or more anxious and it keeps going. (Counsellor Therapist \#2) 
It was also noted that resettlement factors directly impact how mental health can change, not only upon initial arrival whereby many stressors immediately present themselves but also continuously throughout what can be deemed a lifelong resettlement process. After going through what is likely to be a harrowing journey, refugees feel extremely relieved that they have made it to Canada, especially if they are seeking asylum and obtain their Convention refugee status. However, often they are unaware of how many barriers they face until they have been in the country for a certain period of time. For example, one participant explained that a 'bimodal' presentation of mental health challenges exists for refugees whereby:

Different stressors may be very pronounced in the beginning when there's so much chaos and adjustment and they feel overwhelmed... And then some things tend to subside, but as people begin to encounter the real challenges over time of starting their lives over in a new country, which intersects with things like the age that you migrate, the languages that you speak, your race, your gender... your access to socioeconomic resources and social resources, then another wave of stressors and mental health challenges present themselves at different points in time... they say like "I'm just so grateful that I'm safe, I'm so glad that I'm safe". But then a different feeling sets in where they're like "Oh, but I still can't find work and I still can't find proper housing even though I have status now". (Counsellor Therapist \#3).

The process of resettlement is therefore extremely important as the receiving environment and the challenges refugees face have the power to exacerbate or ameliorate psychological distress. As such, resettlement can often result in worse mental health as refugees get caught in a downward spiral due to an intersection of identity dimensions, psychological distress, and unequal social determinants.

Participants moreover highlighted how overcoming resettlement stressors can at least partially mitigate some of the mental health issues that they may dealing with, along with continuing to access mental health supports. One participant discussed a long-standing client who fled his home country due to persecution of both his faith and for the reason that he married someone of a different race. When this client left, his wife was pregnant at the time, and he 
escaped to Canada with the pre-conceived notion that it would be relatively easy for him to bring his wife and child over through family reunification once he had been granted legal status. The participant described his mental health when she initially saw him as incredibly depressed and self-isolating:

He just couldn't even function, he was not functioning. He would be at home, he was renting a room, and basically would leave maybe to just use the bathroom or something very basic like that, but otherwise he would just sleep for something like thirteen to fourteen hours per day. The profound grief and loss of being forced to separate from his wife and child, the anxiety around what is happening with them... the family separation and then the lack of being able to communicate with them just caused him to completely shut down. (Counsellor Therapist \#2)

Regardless of these initial setbacks, he continued to attend counselling and navigated resettlement stressors despite the fact that his wife and child remained in their home country. Although it took a significant amount of time, achieving success in domains such as employment and establishing regular means to communicate with his family abroad had a major impact on his improved mental health outcomes in the longer term.

Similarly, another participant touched on a client who was referred to her because of she had witnessed gross human rights violations resulting from violent conflict in her country of origin. Not surprisingly, the asylum process and waiting for status took an initial toll on her mental health; however, due to language skills, she was not subject to some of the resettlement stressors that exist for other refugees.

She went back to school, she went on to university. She had come from a country that had English as a second language so the language was not a barrier, she was able to speak English which was why she was able to go to university. She got her nursing degree and now she's working. So when she first came, obviously she was extremely down, she was very sad, very depressed, I mean... she was a refugee. (Counsellor Therapist \#1) 
As a result, it was expressed that while this client's slightly less difficult experience of resettlement mitigated some mental health issues, delayed symptoms of trauma were anticipated to surface as time goes on. 


\section{CHAPTER 6: DISCUSSION AND CONCLUSION}

\subsection{Discussion}

The focus of this MRP was to explore the multifaceted aspects of mental health, resettlement, and time amongst refugees in Canada by focusing on the associations between these factors from the understudied perspective of service providers who work with this population. Data points to the importance of the influence contextual factors in the host country have on psychological well-being, taking into consideration those which have particular salience for refugees. Beiser and Hyman (1997) claim that countries of resettlement view their role as offering physical protection for refugees by basing their policies "on an implicit premise that, once admitted to a country of permanent asylum, refugees can and will take care of themselves" (p. 1000). Despite the fact that refugees are, upon arrival in a host country, geographically removed from the persecution which violently uprooted them, the destitute and culturally alien environment that they find themselves in can be its own kind of trauma. Given findings from this study, it is imperative for countries of resettlement to continue to fulfill their humanitarian obligations. This includes not only accepting refugees, but also attending to the interconnected mental health and resettlement needs of refugee populations to ensure that they can enjoy a quality of life in which they risked their lives, endured emotionally devastating events, and underwent a profound amount of loss to achieve. Resettlement to a vastly different country presents significant challenges and barriers for all migrants, but proves to be that much more difficult for refugees due to the intersection of a number of determinants that place them in further marginalized and oppressed positions. Social determinants are the underlying mechanism for the societal, institutional, and individual inequalities that influence their adjustment patterns and contribute to their mental health outcomes in the long term (Kim, 2016). 
It is therefore important to situate changes in refugee mental health within an intersectional social determinants of health framework in order to fully examine how psychosocial and structural mechanisms may act as both contributing and perpetuating factors in the mental health status of refugee populations. English language acquisition was emphasized by participants to be by far the most imperative factor upon resettlement to Canada for their clients, and yet also usually the most challenging due to the way in which language learning is affected by prior trauma, age, and gender. Literature notes that "language proficiency protects mental health by facilitating social contact, by militating against dependence on others, by promoting the development of new social resources, by enlarging the repertoire of individual coping strategies, and by increasing refugees' sense of internal coherence" (Beiser \& Hou, 2001, p. 1323). In opposition, a gap in language proficiency acts as a significant barrier for refugees because it affects almost all other aspects of resettlement, such as access to job opportunities, education, and services, leading to feelings of withdrawal and isolation, confusion, depression, and marginalization (Mawani, 2014). Moreover, language fluency may affect newcomers' willingness to use formal settlement and mental health supports if service providers who communicate in the same language or an interpreter is not readily available (Sadler, 2013).

Participants in this study expanded upon the ways in which separation from key sources of support, such as family, friends, and a familiar community, leads to exceptional stress for refugees. Many of their clients have had to leave children, spouses, and other immediate family members behind in often violent and unsafe countries with the hopes that acquiring refugee status means an unobstructed path to family reunification. Thus, not only are social supports that provide a sense of coherence and coping severed for many refugees, but they live in a constant and intrusive state of fear and guilt, worrying about those they left behind and often unable to 
communicate with them. This further substantiates findings in a study of refugees from Iraq resettled in the United States whereby participants with immediate family member still remaining in Iraq reported more symptoms of PTSD, depression and anxiety, and greater mental health-related disability overall, compared to those without family left behind (Nickerson, Bryant, Steel, Silove, \& Brooks, 2010). The loss of social networks and support for all populations takes a significant toll on mental health; however, the loss of social support becomes even more critical for refugees arriving in a new country (Simich, Beiser, \& Mawani, 2003; Mawani, 2014). Social support and inclusion is particularly salient during stressful life transitions, providing a buffer against reduced well-being by operating not only as a pathway to emotional coping but also as a 'cultural bridge' through shared experience (Simich et al., 2003).

Participants from the current research also expressed that while shared culture within an ethnic or religious community is important for refugees' sense of belonging, a factor important for mental well-being, shared experience within their communities is particularly vital. Literature identifies this as affirmational support, whereby,

Both sociocultural and situational similarity enhance the likelihood of the perception and reception of empathic understanding... Social and value similarities enhance individuals' confidence in their comparative self-evaluations, and such similarities increase perceived applicability of others' experience and guidance. (Thoits, 1986, p. 420)

Thus, literature elaborates that the familiarity and understanding provided by others who are experiencing or have previously experienced similar life experiences is a different type of social support. Service providers in the present study recommended that a useful way to combat stigma surrounding mental health in refugee communities would be to capitalize on the power of shared experience through outreach, having members from specific groups who have struggled with mental health issues provide psychoeducation to their communities. 
Acculturation challenges were also indicated to be a robust factor inherent in mental health. Participants revealed that, while younger generations may have more flexibility in adapting to changes, factors such as age of migration may hinder acculturation for older refugees as they are more firmly rooted in their home culture, face a more difficult time learning a new language, and encounter a greater likelihood of social isolation. In addition, it was expressed that acculturation challenges in post-migration often affects perceptions of the female role, causing changes in family dynamics that can result in relational distress as woman endeavor to navigate their identities and newfound place in a Western context within their cultural and religious milieus. Refugees must therefore negotiate a new, very different environment of the receiving society in comparison with the home they left behind, resulting in a 'dual frame of reference' (Suarez-Orozco, 1997). As articulated by participants, a possible result of these changes, alongside stress resulting from a host of other resettlement factors, can be domestic abuse. This corroborates findings from research set in Sweden by Darvishpour (2002), which examined the relationship between spousal dynamics and mental health in Iranian refugees, finding that spouses are often a source of interpersonal stress rather than an interpersonal resource, with extreme cases resulting in intimate partner violence. Moreover, Beiser (1999) proposes that shifting gender roles heightens a sense of marginalization and depression, particularly among refugee women due to more challenges in navigating new family dynamics. This acculturation stress is compounded by the loss for many refugee women of their professional and personal identity due to both the process of forced migration and government policies that restrict them. Another less recognized but salient dimension of resettlement - undocumented status arose from consensus across all participant interviews as a chronic stressor solely affecting inland refugee claimants. The often long-lasting procedure of filing a claim for asylum and 
undergoing the refugee determination process causes substantial post-migration anxiety for claimants, leading to re-traumatization, exile from being able to properly settle, and feelings of both instability and unsafety. Findings from the current research revealed that refugee claimants may live months or even years in an unstable state of precariousness that primarily revolves around waiting for their hearing or status appeals, acting as a major barrier to participation in everyday society as well as attaining a stable psychological state through addressing prior issues. As confirmed by prior research, participants noted that once status is obtained, feelings of happiness are frequently counteracted by novel resettlement stressors that over time arise and erode refugees' feelings of relief (Silove et al., 2000). This MRP additionally confirms literature which demonstrates that psychopathology decreases upon obtaining secure legal status for refugee claimants (e.g. Drožđek, Kamperman, Tol, Knipscheer, \& Kleber, 2013). Such scholarship concerning the effects of refugee determination procedures on asylum seekers suggest that this reduction in distress is due to feelings of safety and stability as well as relief in gaining legal recognition in a country of resettlement in accordance with Aaron Antonovsky's notion of a 'sense of coherence' (Aviv, 2017). According to Antonovsky, mental health is theorized to depend on the extent to which individuals have pervasive feelings of predictability, structure, and order in their environments, and therefore psychological illness arises from incoherence (Aviv, 2017). Moreover, legal status also plays a crucial role in the lives of forced migrants and, although they may feel initial relief and acceptance in acquiring recognition as a refugee, the presence of many resettlement stressors are dependent on their legality and consequently not entirely realized until this occurs (Drožđek et al., 2013).

Results from the present research study illuminated that not only do challenges in the labour market affect mental health, but they are intensely prominent for male refugees who may 
face a marked difference in socioeconomic position from pre- to post-migration and feel the burden to provide for their families. Kim and Kim (2014) note that unemployment and underemployment has a particular strain on mental health as precarious socioeconomic status in the resettlement context engenders greater pressure on coping resources, triggering negative psychological outcomes. While literature focusing specifically on refugee men is scare, research conducted by Este and Tachble (2008) with Sudanese refugee fathers in Canada found that, despite possible spousal role changes upon resettlement, men viewed their primary role as one of economic provisioning. Thus, as service providers in the current study noted, when refugee men are unable to find jobs in their new environment that accurately reflect their skill, education, and prior socioeconomic level, they express psychological distress due to lowered income compounded by their perceptions of a failure to fulfill this fundamental role. These mental health issues become further exacerbated when the pathways to economic decline include social exclusion and discrimination (Este \& Tachble, 2008).

Refugees are often living at social assistance levels for at least their first year in Canada, with multiple and intersecting oppressions contributing to depression and anxiety coupled with their sense of loss and dislocation. Participants from this study highlighted that mental health for refugees is not seen to be a priority among newcomer services and is consequently largely underfunded. Mukhtar (2013) deconstructs the way in which the public sector is designed, claiming that the allocation of funds to particular groups and certain services illustrate a larger tenet of citizenship building. The state's commitment to provide and fund particular types of settlement support sends the implicit message of who is valued and welcomed in Canada and specifically what type of skills are respected (Root, Gates-Gasse, Shields, \& Bauder, 2014). In this way, the government is able to tacitly define who is entitled to settlement services via the 
allocation of resources (Mukhtar, 2013). Service providers interviewed from the present research indicated that even though refugees are perceived to be low on the priority list comparatively to other migrant groups, such as highly skilled economic immigrants, more funding is provided to refugee focused services that accelerate integration and stress the utilization of human capital. The government-provided services that newcomers encounter upon arrival to Canada hence "reflects the disparate power imbalances between those who are seen as 'Canadian', deserving of and belonging to the nation-state, and those deemed 'others"” (Yee, Marshall, \& Vo, 2014, p. 89). As findings from this MRP reveal, the amount and type of services offered has implications by impacting sense of belonging for refugees as well as combining with language and cultural gaps that complicate mental health care seeking and access. Although the underutilization of 'main-stream' services has been well-documented in the literature, providing another possible explanation for how funds are allocated, the present study supports Kinzie's (1985) contention that refugees will use and benefit from mental health care that is culturally sensitive and appropriate to their needs if it is available. However, the top-down manner in which funding is allocated creates competition within the settlement sector, echoing Watters' (2011) sentiment that " $[\mathrm{t}]$ he context in which refugees receive mental health treatment is crucially influenced by a 'macro-level' in which 'common sense assumptions' regarding their needs construct the very contexts in which services are available" (p. 1711). This further acts as a social determinant, shaping the way that agencies and organizations serving refugees create programs and services in order to receive funding to continue to operate, despite the fact that the statistics extracted from these services may not reflect the bleak reality that they are not assisting the targeted population in the most effective manner. 
The current research has made clear that mental health during the initial periods of resettlement affects subsequent psychological well-being into the medium and longer term for refugees. Earlier studies of mental health specify exposure to traumatic events as an explanation for the high prevalence of psychological illness among refugee populations, both upon initial arrival and over time (e.g. Mollica et al., 1998; Steel et al., 2002), thereby theorizing that prior trauma and stressors "exert a more evanescent mental health effect than contemporaneous factors" (Beiser \& Hyman, 1997, p. 1000). On the contrary, more recent psychoanalytically oriented research demonstrates the effects of resettlement stressors on the persistence and increase in poor mental health (e.g. Miller \& Rasmussen, 2009; Li et al., 2016). Furthermore, the nuanced relationship between pre-migration trauma and the ongoing strain resettlement has upon symptoms of already existing mental distress needs to be considered and analyzed in relation to the passage of time. Findings from the present study are in line with Beiser and Hyman's (1997) proposition that suppression of the past occurs upon resettlement for refugees while more immediate survival needs are being prioritized, as service providers perceived stressors related to immediate settlement needs to have a greater impact on mental health in the short term rather than pre-migration events or trauma that refugees have experienced.

Findings extracted from participant interviews substantiated Grove and Torbiorn's (1985) model, divulging that mental health changes are triggered not only by different stressors that present themselves after the erosion of an initial euphoric period, but also because the phase of resettlement may affect the salience of these stressors. A possible explanation for suppression thus labels it is an adaptive strategy that serves to mitigate mental health symptoms in the aftermath of catastrophic events, leaving mental capacity for the individual to handle survival needs (Beiser \& Hyman, 1997; Miller \& Rasmussen, 2009; Carswell et al., 2009). Nonetheless, 
service providers in this study concluded that, while this may be an adaptive strategy during initial resettlement, long term suppression is unsustainable. Depending on the nature of events during the first two phases of migration, refugees' pasts are likely to re-emerge and threaten mental well-being, indicating a need for those who assist this population to be aware of changing mental health status (Beiser, 1999; Beiser \& Hyman, 1997).

Data from the present study also indicates that service providers with clients who had undergone severe forms of trauma (e.g. torture, rape) exhibited debilitating mental health symptoms upon arrival that consumed their ability to handle even small challenges of resettlement. This results in a mutually reinforcing relationship between post-migration stressors and declining well-being whereby trauma and the resettlement environment are inextricably intertwined. Being disposed, directly or indirectly, to a range of pre-migration atrocities may therefore very well lead to mental health symptoms, some of which mirror the criteria for PTSD. It is the post-migration stressors however that aggravate and trigger these symptoms to a degree that results in decreased functioning and an inability to handle smaller stressors, which in turn makes it that much more difficult for severely traumatized refugees to handle such factors as discrimination, exclusion, and unemployment. As their mental well-being declines, integration for these refugees thus becomes hindered by a sense of personal disarray, and programs designed to serve refugee as a monolithic population are unable to be used due to intersections of disadvantage such as trauma. As elaborated upon earlier, language acquisition has been well documented as one of the significant stressors for refugees. However, because symptoms of trauma have been explained to impact cognitive functioning such as concentration and memory retention, the Language Instruction for Newcomers to Canada program (LINC) that is offered may not be appropriate for refugees suffering from trauma-specific mental health issues. Other 
ways to obtain English skills may be required or not used at all, resulting in the inability to obtain legal employment and access services, lowered self-esteem, and worsened psychological impairment over time. Additionally, those with more severe trauma may experience social anxiety, find it difficult to establish trusting relationships with others, and maintain employment, contributing to a discernible sense of isolation and exacerbated, long-lasting psychological symptoms.

Interviews with mental health service providers revealed that psychological services for refugees need to take on an individualized, client-centered approach that focuses simultaneously on pre- and post-migratory stressors. While more recent scholarship concerned with refugee mental health criticizes that viewpoint that "suppression of past traumata [is] a substrate for the emergence of psychopathology and recovery of the memory of horrific past events a prerequisite to healing" (Beiser \& Hyman, 1997, p. 1001), this study highlights that many refugees are eager and self-motivated to discuss their past trauma with professionals upon arrival in the country of resettlement. A lack of psychoeducation in their home countries perpetuates stigma and may therefore serve as a barrier to accessing services; however, it can also have the opposite effect, with a number of participants observing that many refugees are extremely open to accessing mental health services due to the psychological distress they are aware that they are experiencing but are unable to handle by way of their own resources.

Through the narratives of service providers, this analysis must be expanded to acknowledge not only the unequal distribution of economic and social resources, but also the ability of individuals to mobilize personal resources and how this directly affects mental health. According to stress process theory, experiences that overwhelm quotidian responses jeopardize psychological well-being (Beiser \& Hyman, 1997; Beiser \& Hou, 2001). This model therefore 
proposes that factors such as pre-migration trauma, unemployment or underemployment, social isolation, and other resettlement stressors predispose refugees to mental health issues. However, the ability of refugees to gather and employ personal and social resources, for example learning to speak the language of the host country, building a sense of community, and using coping techniques, at least partially buffers the effects of these stressors (Mawani, 2014). Despite the inequalities and vulnerabilities that refugees face, it was made clear by participants in this study that the motivation to not only survive, but to excel upon arrival in Canada is an attribute many refugees possess. Accordingly, while much of the literature on refugee mental health focuses on the extent to which refugees are subject to and exhibit mental illness due to pre-migration trauma from a pathogenic orientation, findings from this MRP highlight that many refugees cope well despite the numerous barriers they must overcome both before and after resettlement. More attention therefore needs to be directed towards how trauma experienced in the resettlement environment is shaped and influenced by both sociodemographic characteristics and unequal social determinants they encounter upon arrival, and the vital role that resiliency plays in mental health outcomes over time.

\subsection{Limitations and Recommendations for Future Research}

It is of importance to acknowledge several limitations of this research project, in which future directions may address identified gaps or become extensions of the present study. First, Archer and Berdahl (2011) suggest that as many perspectives as possible should be examined in order to best understand a phenomena and increase the 'trustworthiness' of the study. Due to the time restriction of the MRP, this research included a small sample of service providers and a larger sample would have therefore increased the amount and the quality of novel information obtained. Further, while this study incorporated an intersectional lens into the interview process 
as well as through the interpretation of its findings, a distinctly focussed analysis and discussion of refugees from specific countries of origin, genders, and ages who have experienced varying types of trauma may shed light on the relationship between different intersections of multiple identities for refugees and their mental health rather than the somewhat monolithic view of the refugee population that this study offered. This highlights potential directions for future research as the general findings drawn from this study may be transferable to other locales across Canada and refugee mental health issues from the service provider perspective more broadly. An additional issue worth considering is the innumerable factors at play in the multi-faceted complexity that is refugee mental health, which extends beyond the scope of this research in regards to both time and resource constraints. A methodological extension of the present analysis would be a longitudinal study design utilizing a qualitative approach which may reveal richer data by allowing for temporal changes to be assessed at different time points, particularly in regards to the interplay between various social determinants in Canadian society and premigration trauma on mental health.

The asylum process and refugee determination system was a key theme that was discussed by almost every single participant. However, literature is virtually non-existent on the relationship between how specifically the Canadian refugee determination system affects the mental health of refugee claimants, and therefore must be explored in future research. Another key topic was how trauma affects the capacity of some refugees to handle resettlement stressors, such as language acquisition. To date, this has been a vastly understudied yet important topic. In particular, the relationship between different types of trauma, post-migratory stressors such as learning a new language, and the impact on mental health must be further researched. Lastly, future research concerning refugee mental health in Canada may require a focus on particular 
refugee populations along with their service providers in order to provide a juxtaposing examination and comparison of mental health service needs and use.

\subsection{Conclusion}

Migration literature needs to pay more attention to the ways in which intersectional social inequalities are active at all levels that refugees encounter throughout their migration and settlement trajectories. This MRP has attempted to fill some of these gaps and contribute to knowledge on the mental health needs of refugees over time by identifying resettlement stresses that jeopardize mental health, gaining insight into the complex relationship between trauma and post-migration factors, as well as the personal and social resources that help protect against psychological decline. As a result, this study provides an important knowledge base for refugee mental health by suggesting that further innovation in this field needs to take place through an understanding of how psychosocial and structural mechanisms act as both contributing and perpetuating factors in mental health outcomes. This is of importance to highlight because, while Canadian immigration procedures cannot alter the pasts that refugees bring with them, policies and services have the ability to affect post-migration stressors that are, as shown, extremely relevant to mental health outcomes for refugees in all stages of what can be deemed their lifelong resettlement process. 


\section{Appendix A.}

\section{Interview Guide}

1. What is your role and how long have you been working as a mental healthcare service provider for refugees?

2. What mental health services does your agency provide for refugees?

- Which services are the most commonly accessed?

- How long after refugees arrive in Canada do they typically access your mental health services?

- How do refugee clients find out about your services?

3. What are the demographics of the refugee population that you see? (i.e. age, gender, country of origin, marital/family status, etc).

- How do these different intersections of identity affect mental health after resettlement?

4. Research tends to states that for immigrant groups, particularly refugees, mental health services in Canada go underused. What barriers do you think prevent refugees from using mental health services?

5. Which resettlement stressors do you think are the most prevalent in impacting the mental health of your refugee clients?

6. Please take a moment to think about 2-3 past or present clients that you have seen for a longer time (from first arrival in Canada to a number of years) over the course of your career working with refugees. Please do not include any personal or identifying information, such as name and age, of the clients in your answers to the following questions.

- What were their mental health needs upon arrival to Canada and were these needs a priority (or not) during initial resettlement?

- On average, how long did the individual use mental health services?

- How did their mental health needs change after having lived in Canada for a few years from initial arrival? What about over the longer term?

7. How do refugees experience and express mental health issues differently from the Canadian born population that are not refugees?

8. What are some policy or service recommendations that you have to address refugee mental health needs, both upon arrival to Canada and over the medium to long term?

If there is anything else that you feel is important to share or add to this conversation, please do so now. 


\section{Appendix B.}

\section{$\underline{\text { Participant Consent Form }}$}

\section{Ryerson \\ University}

\section{Ryerson University \\ Consent Agreement}

You are being invited to participate in a research study. Please read this consent form so that you understand what your participation will involve. Before you consent to participate, please ask any questions to be sure you understand what your participation will involve. You may take as much time as you wish to decide whether or not to participate in this study.

\section{THE CHANGING MENTAL HEALTH OF REFUGEES OVER TIME}

INVESTIGATORS: This research study is being conducted by:

Stephanie Fraser, M.A.(c) Immigration and Settlement Studies, Ryerson University, for the purposes of a Major Research Paper (MRP) in partial fulfillment of a graduate degree.

If you have any questions or concerns about the research, please feel free to contact:

Stephanie Fraser (Principal Investigator)

stephanie.fraser@ryerson.ca

This research is being conducted under the direct supervision of:

Dr. Sepali Guruge

Department of Nursing

Ryerson University

350 Victoria Street

Toronto, Ontario, M5B 2K3

sguruge@ryerson.ca

416-979-5000 ext. 4964

\section{PURPOSE OF THE STUDY:}

The purpose of this study is to identify the mental health services that refugees require not only upon their initial arrival to Toronto but also over the longer term. The specific research question is: How do the mental health service needs of refugees change over time? 
The outcomes of this research question includes:

- Contribute to the literature concerning refugee mental health.

- Help inform future research with regard to the mental health service needs of refugees.

- To produce research that can help mental health service providers and agencies that serve refugees better assist their clients.

This study is seeking 4-6 mental health care professionals to participate in this research project that are currently employed and have been working with refugee clients for a minimum of three years. I hope that this project will provide a basis for further research and practices for responding to the mental health needs of refugees throughout their resettlement process.

\section{WHAT PARTICIPATION MEANS:}

If you volunteer to participate in this study, you will be asked to participate in a one-on-one interview.

\section{Interview Format:}

- The interview will last 30-45 minutes.

- The interview will be conducted at a mutually convenient time in a booked room at the Student Learning Centre (SLC) at Ryerson University, or at an aurally and visually private place of your choosing.

- You will be provided with all necessary information prior to the interview, including the consent form and possible interview questions.

- I am available to correspond with you before the interview, either by phone or by email. The purpose of this correspondence will be to discuss the nature of the interview and the project, the potential risks and benefits of participation, as well as the means of reducing those risks and for you to ask any questions you may have.

- You are free to stop the interview at any time and to choose not to answer questions.

\section{Type of Information Sought:}

- Subject to your consent, I will collect some personal information, including your name, email address, and phone number.

- Your email address will be collected so that a written transcript of your interview can be returned to you for review.

- You will be given this written transcript within 2 weeks after the interview, and you will have 2 weeks after to provide your feedback, request changes, or withdraw your information or data from the study.

- This study is primarily interested in your experience working as a mental health care professional with the refugee community and how their mental health service needs change over time. 
Retention and Dissemination of Information:

- The interview will be audio-recorded.

- I will make written transcriptions of the interviews, after which the audio recordings will be destroyed.

- You will be provided with access to your transcription, which you may review and revise.

- Transcriptions will be kept for 5 years for the reason that this study may be used for academic conference presentations and/or journal publications at a later date.

- All research findings and publications will be made available to participants upon their request.

- If you would like a copy of the result, please contact me, at stephanie.fraser@ryerson.ca.

\section{POTENTIAL BENEFITS:}

Participants may not benefit directly from the study. However, I hope that the information gained in this study may contribute to literature regarding the changing mental health service needs of refugees over time, thus benefiting professionals and/or agencies who work with refugee clients for the purposes of mental health care. I cannot guarantee, however, that you will receive any benefits from participating in this study.

\section{POTENTIAL RISKS TO YOU AS A PARTICIPANT:}

The risks associated with participation are very low. You may experience discomfort when discussing refugee clients that have sought mental health services from you as well as your own personal experiences working with this population. To reduce this risk, you are not required to answer questions you are uncomfortable with, and the interview can be stopped at any time, either temporarily or permanently. To reduce this risk further, contact information for counselling services can be provided upon your request.

\section{CONFIDENTIALITY:}

I will be collecting some personal information, including your name, email address, and phone number. This is done if I need to contact you after the interview, as well as send you the transcribed audio-recording. I will use pseudonyms so that your name is not attached to the data, and all information that identifies you in the written notes will be removed. Data will be stored separately, in different password-protected files.

There will be no video recordings, and the audio recordings of the interviews will be destroyed once transcribed. You will be given the transcripts to review and revise. Copies of the coded transcriptions will be kept for 5 years in case the study is published or used for other academic purposes. The study results will be made available to you at your request. Transcription of the tapes will be done only by the Principal Investigator.

\section{INCENTIVES FOR PARTICIPATION:}

You may incur travel costs along with time spent in order to be available to take part in this study. You will be provided with $\$ 30$ (thirty dollars) to cover such costs. You will receive this amount even if you decide to withdraw from the study at any time. 


\section{VOLUNTARY PARTICIPATION AND WITHDRAWAL:}

Participation in this study is completely voluntary. You can choose whether to participate in this study or not. If any question makes you uncomfortable, you can skip that question. You may stop participating at any time without penalty or loss of incentives. If you choose to stop participating, you may also choose to not have your data included in the study. After your interview, within 2 weeks you will be given a written transcription for review. You will have 2 weeks once receiving a copy of your transcribed interview to review your responses, request any changes or omissions, or request a full withdrawal from the study. The limited time for withdrawal is due to the relatively short timeline for completion of this study. If you decide to withdraw during this time, we will remove and destroy all of your data from the study. However, after this time period, withdrawal from the study will not result in removal of already collected data. Your choice of whether or not to participate will not influence your future relationship with Ryerson University.

\section{QUESTIONS ABOUT THE STUDY:}

If you have any questions about the research now, please ask. If you have questions later about the research, you may contact:

Stephanie Fraser, M.A.(c) Immigration and Settlement Studies stephanie.fraser@ryerson.ca

Dr. Sepali Guruge

Department of Nursing

Ryerson University 350 Victoria Street

Toronto, Ontario, M5B 2K3

sguruge@ ryerson.ca

416-979-5000 ext. 4964

This study has been reviewed by the Ryerson University Research Ethics Board. If you have questions regarding your rights as a participant in this study, please contact:

Research Ethics Board

c/o Office of the Vice President, Research and Innovation

Ryerson University

350 Victoria Street

Toronto, ON M5B 2K3

416-979-5042

rebchair@ryerson.ca 


\section{References}

Allen, J., Balfour, R., Bell, R., \& Marmot, M. (2014). Social determinants of mental health. International Review of Psychiatry, 26(4), 392-407.

Almqvist, K., \& Broberg, A. G. (1999). Mental health and social adjustment in young refugee children three and a half years after their arrival in Sweden. Journal of the American Academy of Child \& Adolescent Psychiatry, 38(6), 723-730.

Archer, K., \& Berdahl, L. (2011). Explorations: Conducting Empirical Research in Canadian Political Science, Second Edition. Don Mills, ON: Oxford University Press.

Asaam, H. (2015). Mental health and well-being of refugees to Canada: A brighter future (Master's Thesis). University of Manitoba, Winnipeg.

Aviv, R. (2017). The Trauma of Facing Deportation. The New Yorker. Retrieved from http://www.newyorker.com/magazine/2017/04/03/the-trauma-of-facing-deportation

Bartolomei, L., Pittaway, E., \& Pittaway, E.E. (2003). Who am I?: Identity and citizenship in Kakuma refugee camp in northern Kenya. Development, 46(3), 87-93

Beiser, M. (1988). After the Door has been opened-mental health issues affecting immigrants and refugees in Canada: Report of the Canadian Task Force on Mental Health Issues Affecting Immigrants and Refugees.

Beiser, M. (1999). Strangers at the gate: The 'boat people's' fist ten years in Canada. Toronto: University of Toronto Press.

Beiser, M. N., \& Hou, F. (2006). Ethnic identity, resettlement stress and depressive affect among Southeast Asian refugees in Canada. Social Science \& Medicine, 63(1), 137-150.

Beiser, M., \& Hyman, I. (1997). Refugees' time perspective and mental health. American Journal of Psychiatry, 154(7), 996-1002.

Beiser, M., \& Wickrama, K. (2004). Trauma, time and mental health: A study of temporal reintegration and Depressive Disorder among Southeast Asian refugees. Psychological Medicine, 34(05), 899-910.

Berg, B.L. (2001). Qualitative Research Methods for the Social Sciences. Needham Heights: A Pearson Education Company.

Bhugra, D. (2004). Migration and mental health. Acta Psychiatrica Scandinavica, 109(4), 243258.

Braun, V., \& Clarke, V. (2006). Using thematic analysis in psychology. Qualitative research in psychology, 3(2), 77-101. 
Bryman, A. (2001). Social Research Methods. Oxford: Oxford University Press.

Carswell, K., Blackburn, P., \& Barker, C. (2009). The relationship between trauma, postmigration problems and the psychological well-being of refugees and asylum seekers. International Journal of Social Psychiatry, 57(2), 107-119.

Citizenship and Immigration Canada (2013). Terms and definitions related to refugee protection. Retrieved from http://www.cic.gc.ca/english/resources/tools/refugees/definitions.asp

Craig, C. D., Sossou, M. A., Schnak, M., \& Essex, H. (2008). Complicated grief and its relationship to mental health and well-being among Bosnian refugees after resettlement in the United States: Implications for practice, policy, and research. Traumatology, 14(4), 103.

Crenshaw, K. (1989). Demarginalizing the intersection of race and sex: A black feminist critique of antidiscrimination doctrine, feminist theory and antiracist politics. U. Chi. Legal F., 139.

Creswell, J. W., Hanson, W. E., Plano Clark, V. L., \& Morales, A. (2007). Qualitative research designs: Selection and implementation. The counseling psychologist, 35(2), 236-264.

Deng, S. A., \& Marlowe, J. M. (2013). Refugee resettlement and parenting in a different context. Journal of Immigrant \& Refugee Studies, 11(4), 416-430.

Drožđek, B., Kamperman, A. M., Tol, W. A., Knipscheer, J. W., \& Kleber, R. J. (2013). Is legal status impacting outcomes of group therapy for posttraumatic stress disorder with male asylum seekers and refugees from Iran and Afghanistan?. BMC psychiatry, 13(1), 148.

Elliott, D., \& Segal, U. A. (2012). Refugees worldwide. Praeger.

Elo, S., \& Kyngäs, H. (2008). The qualitative content analysis process. Journal of advanced nursing, 62(1), 107-115.

Este, D. C., \& Tachble, A. (2009). Fatherhood in the Canadian context: Perceptions and experiences of Sudanese refugee men. Sex Roles, 60(7-8), 456-466.

Fazel, M., Wheeler, J., \& Danesh, J. (2005). Prevalence of serious mental disorder in 7000 refugees resettled in Western countries: A systematic review. The Lancet, 365(9467), $1309-1314$.

Forman, J., \& Damschroder, L. (2007). Qualitative content analysis. In Empirical methods for bioethics: A primer (pp. 39-62). Emerald Group Publishing Limited.

George, M. (2012). Migration traumatic experiences and refugee distress: Implications for social work practice. Clinical Social Work Journal, 40(4), 429-437. 
Gonsalves, C. J. (1992). Psychological stages of the refugee process: A model for therapeutic interventions. Professional Psychology: Research and Practice, 23(5), 382.

Guruge, S., \& Khanlou, N. (2004). Intersectionalities of influence: Researching the health of immigrant and refugee women. CJNR (Canadian Journal of Nursing Research), 36(3), 3247.

Gushulak, B. D., Pottie, K., Roberts, J. H., Torres, S., \& DesMeules, M. (2011). Migration and health in Canada: health in the global village. Canadian Medical Association Journal, 183(12), E952-E958.

Hadley, C., \& Patil, C. (2009). Perceived discrimination among three groups of refugees resettled in the USA: Associations with language, time in the USA, and continent of origin. Journal of immigrant and minority health, 11(6), 505.

Haldane, J., \& Nickerson, A. (2016). The impact of interpersonal and noninterpersonal trauma on psychological symptoms in refugees: The moderating role of gender and trauma type. Journal of traumatic stress, 29(5), 457-465.

Hallett, K. (2010). Intersectionality and serious mental illness - A case study and recommendations for practice. Women \& Therapy, 38(1-2), 156-174.

Hayes, M.V., \& Dunn, J.R. (1998). Population health in Canada: A systematic review. Ottawa: Canadian Policy Research Network (CPRN).

Hodes, M. (2000). Psychologically distressed refugee children in the United Kingdom. Child and Adolescent Mental Health, 5(2), 57-68.

Hollander, A. C. (2013). Social inequalities in mental health and mortality among refugees and other immigrants to Sweden. Global health action, 6(1), 21059.

Ingleby, D. (Ed.). (2004). Forced migration and mental health: Rethinking the care of refugees and displaced persons. Springer Science \& Business Media.

Ip, C.K.C. (2016). Examining mental health status and mental health service utilization by visible minority immigrants in Canada: Adopting a social determinants of health and intersectionality approach. Master's Thesis. University of Calgary, Alberta.

Iyer, A., Sen, G., \& Östlin, P. (2008). The intersections of gender and class in health status and health care. Global Public Health, 3(S1), 13-24.

Kim, I. (2016). Beyond trauma: post-resettlement factors and mental health outcomes among Latino and Asian refugees in the United States. Journal of Immigrant and Minority Health, 18(4), 740-748. 
Kim, I., Chen, J., \& Spencer, M. S. (2012). Social determinants of health and mental health among Asian Americans in the United States. Journal of the Society for Social Work and Research, 3(4), 346-361.

Kim, I., \& Kim, W. (2014). Post-resettlement challenges and mental health of Southeast Asian Refugees in the United States. Best Practices in Mental Health, 10(2), 63-77.

Kinzie, J. D. (1985). Cultural aspects of psychiatric treatment with Indochinese refugees. American Journal of Social Psychiatry, 14, 105-111.

Kirmayer, L. J., Groleau, D., Guzder, J., Blake, C., \& Jarvis, E. (2003). Cultural consultation: A model of mental health service for multicultural societies. The Canadian Journal of Psychiatry, 48(3), 145-153.

Lacroix, M. (2004). Canadian refugee policy and the social construction of the refugee claimant subjectivity: Understanding refugeeness. Journal of refugee studies, 17(2), 147-166.

Lacroix, M., \& Sabbah, C. (2011). Posttraumatic psychological distress and resettlement: The need for a different practice in assisting refugee families. Journal of Family Social Work, 14(1), 43-53.

Lamb, C. F., \& Smith, M. (2002). Problems refugees face when accessing health services. New South Wales public health bulletin, 13(7), 161-163.

Langer, B. (1990). The continuing trauma of refugee settlement - The experience of El Salvadoreans. In P. Hosking (ed). Hope After Horror Sydney, Uniya.

LeMaster, J. W., Broadbridge, C. L., Lumley, M. A., Arnetz, J. E., Arfken, C., Fetters, M. D., ... \& Arnetz, B. B. (2017). Acculturation and Post-Migration Psychological Symptoms Among Iraqi Refugees: A Path Analysis. The American journal of orthopsychiatry.

Lie, B. (2002). A 3-year follow-up study of psychosocial functioning and general symptoms in settled refugees. Acta Psychiatrica Scandinavica, 106(6), 415-425.

Makwarimba, E., Stewart, M., Simich, L., Makumbe, K., Shizha, E., \& Anderson, S. (2013). Sudanese and Somali refugees in Canada: Social support needs and preferences. International Migration, 51(5), 106-119.

Marmot, M., \& Wilkinson, R. G. (2006). Social determinants of health ( $2^{\text {nd }}$ ed.). New York: Oxford University Press.

Marsella, A. J., Bornemann, T. E., Ekblad, S. E., \& Orley, J. E. (1994). Amidst peril and pain: The mental health and well-being of the world's refugees. American Psychological Association. 
Marshall, G. N., Schell, T. L., Elliott, M. N., Berthold, S. M., \& Chun, C. A. (2005). Mental health of Cambodian refugees two decades after resettlement in the United States. Jama, 294(5), 571-579.

Mawani, F. N. (2014). Social determinants of refugee mental health. In Refuge and Resilience (pp. 27-50). Springer Netherlands.

Meadows, L. M., Thurston, W. E., \& Melton, C. (2001). Immigrant women's health. Social science \& medicine, 52(9), 1451-1458.

Miller, K. E., \& Rasco, L. M. (2004). The mental health of refugees: Ecological approaches to healing and adaptation. Taylor \& Francis.

Miller, K. E., \& Rasmussen, A. (2010). War exposure, daily stressors, and mental health in conflict and post-conflict settings: Bridging the divide between trauma-focused and psychosocial frameworks. Social science \& medicine, 70(1), 7-16.

Morgan, A., \& Ziglio, E. (2007). Revitalising the evidence base for public health: An assets model. Promotion \& Education, 14(2), 17-22.

Mukhtar, M. (2013). Settlement service providers in Peel Region, Ontario: Challenges, barriers and opportunities in the shadow state. (Doctoral dissertation, University of Toronto).

Murray, K. E., Davidson, G. R., \& Schweitzer, R. D. (2010). Review of refugee mental health interventions following resettlement: best practices and recommendations. American Journal of Orthopsychiatry, 80(4), 576-585.

Murray, S. B., \& Skull, S. A. (2005). Hurdles to health: immigrant and refugee health care in Australia. Australian Health Review, 29(1), 25-29.

Nickerson, A., Bryant, R. A., Steel, Z., Silove, D., \& Brooks, R. (2010). The impact of fear for family on mental health in a resettled Iraqi refugee community. Journal of psychiatric research, 44(4), 229-235.

Nickerson, A., Bryant, R. A., Silove, D., \& Steel, Z. (2011). A critical review of psychological treatments of posttraumatic stress disorder in refugees. Clinical psychology review, 31(3), 399-417.

Patton M.Q. (1990) Qualitative Evaluation and Research Methods. Sage, Newbury Park, California.

Patton, M. Q. (2002). Two decades of developments in qualitative inquiry a personal, experiential perspective. Qualitative social work, 1(3), 261-283. 
Porter, M., \& Haslam, N. (2005). Predisplacement and postdisplacement factors associated with mental health of refugees and internally displaced persons: A meta-analysis. Jama, 294(5), 602-612.

Raphael, D. (2009). Social determinants of health: an overview of key issues and themes. In D. Raphael, Social determinants of health: Canadian perspectives ( $2^{\text {nd }}$ ed., pp. 2-19). Toronto: Canadian Scholars' Press Inc.

Root, J., E. Gates-Gasse, J. Shields, \& Bauder, H. (2014). Discounting immigrant families: Neoliberalism and the framing of Canadian immigration policy change. Ryerson Centre for Immigration \& Settlement (RCIS) Working Paper.

Sadler, L. (2013). Overcoming Barriers to Post-Secondary Education for Karen Refugees in Langley and Surrey BC. University of Victoria Prepared for the Centre for Interdisciplinary Research: Community Learning and Engagement (CIR:CLE)

Sandelowski, M. (2000). Focus on research methods-whatever happened to qualitative description?. Research in nursing and health, 23(4), 334-340.

Savic, M., Chur-Hansen, A., Mahmood, M. A., \& Moore, V. M. (2016). 'We don't have to go and see a special person to solve this problem': Trauma, mental health beliefs and processes for addressing 'mental health issues' among Sudanese refugees in Australia. International Journal of Social Psychiatry, 62(1), 76-83.

Silove, D. (1999). The psychosocial effects of torture, mass human rights violations, and refugee trauma: Toward an integrated conceptual framework. The Journal of nervous and mental disease, 187(4), 200-207.

Silove, D., Steel, Z., \& Watters, C. (2000). Policies of deterrence and the mental health of asylum seekers. Jama, 284(5), 604-611.

Simich, L., Este, D., \& Hamilton, H. (2010). Meanings of home and mental well-being among Sudanese refugees in Canada. Ethnicity \& health, 15(2), 199-212.

Steel, Z., Silove, D., Phan, T., \& Bauman, A. (2002). Long-term effect of psychological trauma on the mental health of Vietnamese refugees resettled in Australia: a population-based study. The Lancet, 360(9339), 1056-1062.

Steel, Z., Silove, D., Bird, K., McGorry, P., \& Mohan, P. (1999). Pathways from war trauma to posttraumatic stress symptoms among Tamil asylum seekers, refugees, and immigrants. Journal of traumatic stress, 12(3), 421-435.

Suarez-Orozco, M. M. (1997). The cultural psychology of immigration. Health and social services among international labor migrants: A comparative perspective, 131-150. 
Thoits, P. A. (1986). Social support as coping assistance. Journal of consulting and clinical psychology, 54(4), 416.

Thomas, S., Nafees, B. and Bhugra, D. (2004) “"I was running away from death" - The preflight experiences of unaccompanied asylum seeking children in the UK. Child care health and development, 30(2), 113-22.

Thorne, S., Kirkham, S.R., \& O'Flynn-Magee, K. (2004). The analytic challenge in interpretive description. International journal of qualitative methods, 3(1), 1-11.

Vaismoradi, M., Turunen, H., \& Bondas, T. (2013). Content analysis and thematic analysis: Implications for conducting a qualitative descriptive study. Nursing \& health sciences, 15(3), 398-405.

Van den Hoonaard, D. K. (2012). Qualitative research in action: A Canadian primer. Oxford University Press Canada.

Watters, C. (2001). Emerging paradigms in the mental health care of refugees. Social Science \& Medicine, 52(11), 1709-1718.

Wessels, W. (2014) The refugee experience: Involving pre-migration, in transit, and post migration issues in social services. Master of Social Work Clinical Research Papers. Retrieved from http://sophia.stkate.edu/msw papers/409.

Westermeyer, J., Neider, J., \& Callies, A. (1989). Psychosocial adjustment of Hmong refugees during their first decade in the United States: a longitudinal study. The Journal of nervous and mental disease, 177(3), 132-139.

Whittaker, A. (2009). Research Skills for Social Work. Exeter: Sage Publications, Ltd. UK.

Wilkinson, R. G., \& Marmot, M. (2003). Social determinants of health: The solid facts. World Health Organization.

Wilson, R. M., Murtaza, R., \& Shakya, Y. B. (2010). Pre-migration and post-migration determinants of mental health for newly arrived refugees in Toronto. Canadian Issues, 45.

Wolcott, H. F. (1994). Transforming qualitative data: Description, analysis, and interpretation. Sage. 